\title{
Hopf algebra structure of generalized quasi-symmetric functions in partially commutative variables
}

\author{
Adam Doliwa* \\ Faculty of Mathematics and Computer Science \\ University of Warmia and Mazury \\ ul. Słoneczna 54, 10-710 Olsztyn, Poland \\ doliwa@matman.uwm.edu.pl
}

Submitted: Jan 20, 2021; Accepted: May 17, 2021; Published: Jun 18, 2021

(C) The author. Released under the CC BY-ND license (International 4.0).

\begin{abstract}
We introduce a coloured generalization $\mathrm{NSym}_{A}$ of the Hopf algebra of noncommutative symmetric functions described as a subalgebra of the of rooted ordered coloured trees Hopf algebra. Its natural basis can be identified with the set of sentences over alphabet $A$ (the set of colours). We present also its graded dual algebra $\mathrm{QSym}_{A}$ of coloured quasi-symmetric functions together with its realization in terms of power series in partially commutative variables. We provide formulas expressing multiplication, comultiplication and the antipode for these Hopf algebras in various bases - the corresponding generalizations of the complete homogeneous, elementary, ribbon Schur and power sum bases of NSym, and the monomial and fundamental bases of QSym. We study also certain distinguished series of trees in the setting of restricted duals to Hopf algebras.
\end{abstract}

Mathematics Subject Classifications: 05E05, 16T30, 05C25, 06A07, 68R15

\section{Introduction}

Theory of Hopf algebras forms a modern basis for understanding symmetries of solvable models in quantum and statistical theoretical physics [55, 45, 12]. Application of Hopf algebras $[1,74]$ to combinatorics can be traced back to Rota [43]; see also [70, 34] for more recent reviews of the subject, which has expanded since then. Combinatorial aspects of the Bethe ansatz and of the quantum inverse scattering method [47] were studied, for example, in works by Fomin, Kirillov and Reshetikhin [31, 44]. For more

\footnotetext{
*Supported by NSC grant 2015/19/B/ST2/03575.
} 
about mutual interactions between the theory of integrable systems and combinatorics, see recent reviews $[14,36,16,78]$.

Hopf algebras of rooted trees appeared in the analysis of Runge-Kutta methods by Butcher [10] and Dür [24], and in works by Grossman and Larson [35] in the context of symbolic computation. More recently they have been used by Connes and Kreimer [13] to describe renormalization procedure of quantum field theory; see also [8, 9]. The noncommutative Hopf algebra of trees and forests, generalizing that of Connes and Kreimer, was considered by Foissy [29], and independently by Holtkamp [40].

The theory of symmetric functions $[72,52]$ is by now a well established subject with numerous applications in algebraic topology, combinatorics, representation theory, integrable systems and geometry. Quasi-symmetric functions, introduced by Gessel [33] (see also an earlier relevant work of Stanley [71]), are extensions of symmetric functions that are becoming of comparable importance $[51,3,58]$. As a graded Hopf algebra, the dual of the algebra of quasi-symmetric functions is the Hopf algebra of non-commutative symmetric functions introduced by Gelfand, Krob, Lascoux, Leclerc, Retakh and Thibon [32]. In the works of Zhao [77] and Hoffman [39] an isomorphism has been established between the Hopf algebra of non-commutative symmetric functions and certain subalgebra of rooted ordered trees; see also [25] for application of such trees (called ladders) to study integrable aspect of the renormalization.

Our work arose from the search for generalization of the relationship between theory of symmetric functions, combinatorics and the integrable systems on the non-commutative level. The standard description of the Kadomtsev-Petviashvili (KP) hierarchy of integrable partial differential equations in terms of free fermions by the Kyoto School [62] already involves a large part of the theory of symmetric functions [69]; see also a generalization [17] in direction of quasi-symmetric functions. For example, the Schur functions when expressed in suitably scaled power sum functions (times of the KP hierarchy) provide polynomial $\tau$-function solutions of the equations. Non-commutative extensions of integrable systems are of growing interest in mathematical physics $[49,7,63,28,15,46,18,20,22,21]$.

In this paper we define and study properties of a coloured version of the Hopf algebra of non-commutative symmetric function and of its graded dual. The idea to consider coloured versions of various algebras is not new, see for example [2, 41, 42, 57, 65, 64, 66], where some generalizations of the Hopf algebras of non-commutative symmetric functions or quasi-symmetric functions have been discussed as well. The generalization presented in our work is to our best knowledge new, and in particular it extends some of the previous concepts, see the last remark of Section 3.1.

Let us present the structure of the paper, where we give our generalization of the basic structural elements of the theory of quasi-symmetric and non-commutative symmetric functions showing similarities and differences with the original theory. In introductory Section 2 we recall necessary elements of the theory of graded Hopf algebras. As basic examples we take the free Hopf algebra over finite alphabet, the Hopf algebra of quasi-symmetric functions, and their duals - the shuffle algebra and the algebra of noncommutative symmetric functions. We also pay special attention to the Hopf algebra of rooted ordered coloured trees, closely related to the algebraic renormalization procedure 
of the quantum field theory.

Then in Section 3, we study the Hopf algebra of sentences (coloured compositions or tall trees), the partial order in the set of sentences, and we briefly provide an interpretation of the sentences as words of certain basic context-free language. Then we slightly change our point of view by presenting the sentences over an alphabet $A$ as the analog of the complete homogeneous basis in our coloured non-commutative generalization $\mathrm{NSym}_{A}$ of the Hopf algebra of symmetric functions. Then we introduce the corresponding analog of the basis of elementary functions and discuss coloured version of the standard formulas describing mutual interrelation between the complete homogeneous and elementary functions.

We devote Section 4 to a description of the Hopf algebra QSym $_{A}$ of coloured quasisymmetric functions as graded dual of $\mathrm{NSym}_{A}$. We first define the basis of coloured monomial quasi-symmetric functions in the standard way as the dual basis to the coloured complete homogeneous functions. Then we construct its 'polynomial' realization in terms of certain power series of bounded degree in partially commuting variables. Such a partial commutativity is completely new ingredient of our generalization of the theory of quasi-symmetric functions. We remark that partially commutative variables have been introduced to study combinatorial problems by Cartier and Foata in [11]. They have also found applications in algebra, theory of orthogonal polynomials, statistical physics and computer science; see review by Viennot [76] written in terms of heaps of pieces. In theoretical computer science, as proposed by Mazurkiewicz [59], they describe concurrent computations. We would like to stress that both algebras $\mathrm{NSym}_{A}$ and $\mathrm{QSym}_{A}$ are non-commutative and non-cocommutative for $|A|>1$.

Sections 5 and 6 are devoted to a presentation of the coloured generalization of other rudimentary elements of the theory of symmetric functions. We define and study the fundamental basis of QSym ${ }_{A}$ and its dual basis in $\mathrm{NSym}_{A}$ of coloured non-commutative ribbon Schur functions. Finally we construct the coloured non-commutative version of the power sum symmetric functions. In Section 6 we also extend the algebra of trees to its infinite series version working within the setting of the restricted dual Hopf algebras. This point of view is useful in studying the coloured non-commutative power sum functions, but certainly deserves deeper studies in the context of applications of combinatorial Hopf algebras to integrable systems and theoretical physics.

\section{Hopf algebras of trees and quasi-symmetric functions}

We assume that the Reader is familiar with the basic definitions and properties of Hopf algebras, as covered in [1] or [74]. All the results presented in this Section are known, but we recall them to provide necessary terminology and background to formulate new ones in the next Sections. In the paper all algebras are over a fixed field $\mathbb{k}$ of characteristic zero, although sometimes a commutative ring may be enough. 


\subsection{Hopf algebras}

By $(\mathcal{H}, \mu, \eta, \Delta, \epsilon)$ denote a bialgebra which is:

1. an associative algebra $(\mathcal{H}, \mu, \eta)$ consisting of $\mathbb{k}$-linear multiplication $\mu: \mathcal{H} \otimes \mathcal{H} \rightarrow \mathcal{H}$ and $\mathbb{k}$-linear unit map $\eta: \mathbb{k} \rightarrow \mathcal{H}$ satisfying properties described by the diagrams:

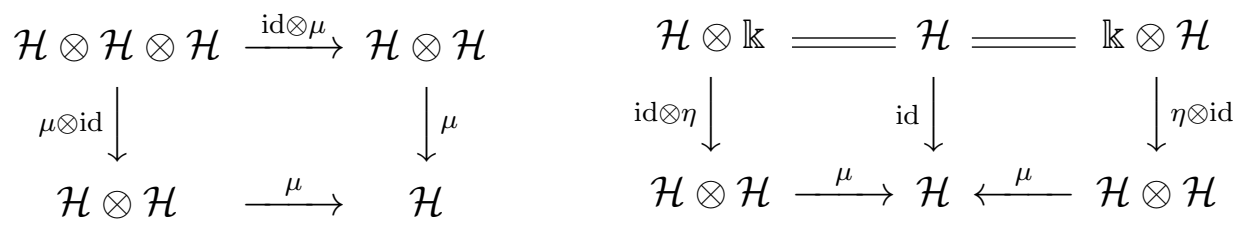

2. a co-associative coalgebra $(\mathcal{H}, \Delta, \epsilon)$ consisting of $\mathbb{k}$-linear comultiplication $\Delta: \mathcal{H} \rightarrow$ $\mathcal{H} \otimes \mathcal{H}$ and $\mathbb{k}$-linear counit map $\epsilon: \mathcal{H} \rightarrow \mathbb{k}$ satisfying properties described by the diagrams:
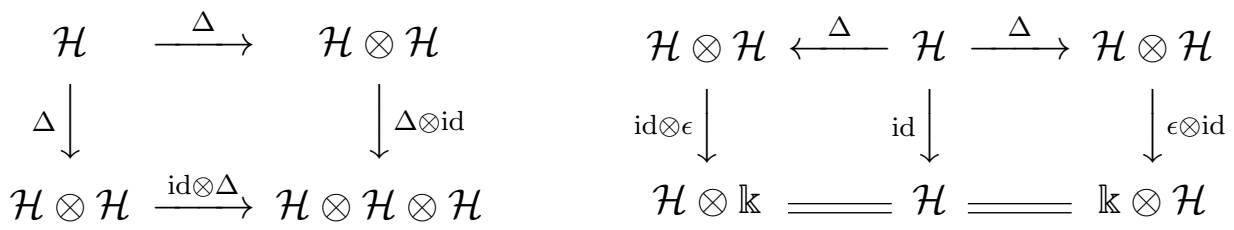

3. such that $\Delta: \mathcal{H} \rightarrow \mathcal{H} \otimes \mathcal{H}$ and $\epsilon: \mathcal{H} \rightarrow \mathbb{k}$ are unital algebra morphisms.

An element $x / y$ of a bialgebra is called primitive/group-like if

$$
\Delta(x)=1 \otimes x+x \otimes 1 \quad / \quad \Delta(y)=y \otimes y .
$$

Bialgebra $\mathcal{H}$ is graded if it is graded as $\mathbb{k}$-module $\mathcal{H}=\bigoplus_{n \geqslant 0} \mathcal{H}^{(n)}$ with the structure maps respecting the gradation

$$
\mathcal{H}^{(n)} \otimes \mathcal{H}^{(m)} \stackrel{\mu}{\rightarrow} \mathcal{H}^{(n+m)}, \quad \mathcal{H}^{(n)} \stackrel{\Delta}{\rightarrow} \bigoplus_{n^{\prime}+n^{\prime \prime}=n} \mathcal{H}^{\left(n^{\prime}\right)} \otimes \mathcal{H}^{\left(n^{\prime \prime}\right)}
$$

A graded bialgebra is connected if $\mathcal{H}^{(0)} \cong \mathbb{k}$.

The space of $\mathbb{k}$-linear operators $\operatorname{End}(\mathcal{H})$ can be equipped with the convolution product $\star: \operatorname{End}(\mathcal{H}) \otimes \operatorname{End}(\mathcal{H}) \rightarrow \operatorname{End}(\mathcal{H})$ defined for $f, g \in \operatorname{End}(\mathcal{A})$ as follows

$$
f \star g=\mu \circ(f \otimes g) \circ \Delta .
$$

Such a product is associative with neutral element $\eta \circ \epsilon$. A bialgebra $\mathcal{H}$ is called a $H o p f$ algebra if there is an element $S \in \operatorname{End}_{\mathbb{k}}(\mathcal{H})$, called antipode, which is two-sided inverse under $\star$ for the identity map id ${ }_{\mathcal{H}}$, which means

$$
\operatorname{id}_{\mathcal{H}} \star S=S \star \operatorname{id}_{\mathcal{H}}=\eta \circ \epsilon .
$$

When it exists, the antipode $S$ is unique and is algebra anti-endomorphism: $S(1)=1$, and $S(a b)=S(b) S(a)$ for all $a, b \in \mathcal{H}$. It is known [60,75] that any graded connected bialgebra 
is a Hopf algebra. In that case the antipode of any homogeneous element $x \in \mathcal{H}^{(n)}$ of degree $n>0$ can be calculated recursively by

$$
S(x)=-x-\sum_{i} S\left(y_{i}\right) z_{i}=-x-\sum_{i} y_{i} S\left(z_{i}\right)
$$

where

$$
\Delta x=1 \otimes x+\sum_{i} y_{i} \otimes z_{i}+x \otimes 1,
$$

and $y_{i}, z_{i}$ have degrees less then $n$.

Example 1. Let $A=\left\{a_{1}, \ldots, a_{m}\right\}$ be a finite set, called an alphabet, whose elements will be called letters. A finite sequence of letters is called a word. The set of all words on $A$ is denoted by $A^{*}$ (the Kleene closure operation $*$ used here shouldn't be confused with the duality sign) and turns out to be free monoid with the concatenation product (denoted by dot "." but usually omitted). The empty sequence plays the role of the neutral element of multiplication and will be denoted by 1 . Consider free algebra $\mathbb{k}\langle A\rangle=\left(\mathbb{k} A^{*}\right.$, . ), whose linear basis consists of words, and the multiplication is given by concatenation of words, extended by linearity.

The unique compatible comultiplication and counit in $\mathbb{k}\langle A\rangle$ is given on letters $a_{i} \in A$ by

$$
\Delta\left(a_{i}\right)=1 \otimes a_{i}+a_{i} \otimes 1, \quad \epsilon\left(a_{i}\right)=0, \quad \forall a_{i} \in A,
$$

and extended by homomorphism to words and by linearity to the whole algebra. Given word $w=a_{i_{1}} \ldots a_{i_{n}}$ we have then

$$
\Delta\left(a_{i_{1}} \ldots a_{i_{n}}\right)=\sum_{J \subset\left(i_{1}, i_{2}, \ldots, i_{n}\right)} w_{J} \otimes w_{\bar{J}}
$$

where the multiindex $J=\left(j_{1}, j_{2}, \ldots, j_{k}\right)$ is a subsequence of $\left(i_{1}, i_{2}, \ldots, i_{n}\right)$, which defines the corresponding word $w_{J}=\left(a_{j_{1}} a_{j_{2}} \ldots a_{j_{k}}\right)$, and $w_{\bar{J}}$ is defined analogously for the complementary subsequence $\bar{J}$. The algebra is cocommutative, graded with gradation being the length of words $\left|a_{i_{1}} \ldots a_{i_{n}}\right|=n$, locally finite and connected. The antipode on words reads $S\left(a_{i_{1}} \ldots a_{i_{n}}\right)=(-1)^{n} a_{i_{n}} \ldots a_{i_{1}}$.

Two Hopf $\mathbb{k}$-algebras $\mathcal{A}, \mathcal{B}$ are dually paired by a map $\langle\rangle:, \mathcal{B} \otimes \mathcal{A} \rightarrow \mathbb{k}$ if

$$
\begin{gathered}
\left\langle\mu_{\mathcal{B}}\left(b_{1}, b_{2}\right), a\right\rangle=\left\langle b_{1} \otimes_{\mathcal{B}} b_{2}, \Delta_{\mathcal{A}}(a)\right\rangle, \quad\left\langle 1_{\mathcal{B}}, a\right\rangle=\epsilon_{\mathcal{A}}(a), \\
\left\langle\Delta_{\mathcal{B}}(b), a_{1} \otimes_{\mathcal{A}} a_{2}\right\rangle=\left\langle b, \mu_{\mathcal{A}}\left(a_{1}, a_{2}\right)\right\rangle, \quad \epsilon_{\mathcal{B}}(b)=\left\langle b, 1_{\mathcal{A}}\right\rangle \\
\left\langle S_{\mathcal{B}}(b), a\right\rangle=\left\langle b, S_{\mathcal{A}}(a)\right\rangle
\end{gathered}
$$

which is then extended to tensor products pairwise. This means that the product of $\mathcal{A}$ and coproduct of $\mathcal{B}$ are adjoint to each other under $\langle$,$\rangle , and vice-versa. Likewise, the$ units and counits are mutually adjoint, and the antipodes are adjoint. In such case any subalgebra of $\mathcal{A}$ gives rise to the corresponding quotient algebra in $\mathcal{B}$. 
When the Hopf algebra $\mathcal{H}$ is finite dimensional then the natural pairing between the $\mathbb{k}$-module $\mathcal{H}$ and its dual $\mathcal{H}^{*}$ allows to introduce on the latter the dual Hopf algebra structure. When $\mathcal{H}$ is infinite dimensional then there is no such general construction, which is caused by the fact that the inclusion $\mathcal{H}^{*} \otimes \mathcal{H}^{*} \subset(\mathcal{H} \otimes \mathcal{H})^{*}$ fails to be equality. For connected graded Hopf algebra $\mathcal{H}=\bigoplus_{n \geqslant 0} \mathcal{H}^{(n)}$ which is locally finite (each homogeneous component $\mathcal{H}^{(n)}$ is finite dimensional), one can define its graded dual as $\mathcal{H}^{g r}=\bigoplus_{n \geqslant 0} \mathcal{H}^{(n) *}$ which has the property that $\mathcal{H}^{g r} \otimes \mathcal{H}^{g r}=(\mathcal{H} \otimes \mathcal{H})^{g r}$ and $\left(\mathcal{H}^{g r}\right)^{g r} \cong \mathcal{H}$. Then $\mathcal{H}^{g r} \subset \mathcal{H}^{*}$ is a Hopf algebra where the evaluation map $\mathcal{H}^{g r} \otimes \mathcal{H} \rightarrow \mathbb{k}$ provides a duality pairing of $\mathcal{H}$ with $\mathcal{H}^{g r}$.

Remark 2. In Section 6 we will consider also another construction of a dual Hopf algebra, called the restricted (or Sweedler's) dual [1,74].

Example 3. The graded dual to the Hopf free algebra $\mathbb{k}\langle A\rangle$ is described as follows. By standard abuse of notation one identifies a fixed linear basis of a finite dimensional space with its dual. The dual (deconcatenation) coproduct $\delta$ is given on words by

$$
\delta\left(a_{i_{1}} \ldots a_{i_{n}}\right)=\sum_{k=0}^{n} a_{i_{1}} \ldots a_{i_{k}} \otimes a_{i_{k+1}} \ldots a_{i_{n}} .
$$

The corresponding product $\sqcup$ (called shuffle product) dual to the coproduct $\Delta$ is given by

$$
a_{i_{1}} \ldots a_{i_{k}} \uplus a_{j_{1}} \ldots a_{j_{l}}=\sum_{I=\left(k_{1}, k_{2}, \ldots, k_{n}\right)} a_{k_{1}} a_{k_{2}} \ldots a_{k_{n}},
$$

where summation is over all sequences $I=\left(k_{1}, k_{2}, \ldots, k_{n}\right)$ such that $\left(i_{1}, i_{2}, \ldots, i_{k}\right) \subset I$ is its subsequence, and $\left(j_{1}, j_{2}, \ldots, j_{l}\right)$ is the complementary subsequence. The unit, counit and antipode in the graded dual are the same as in the previous example.

Remark 4. It is known [50] that the shuffle product of words can be defined recursively for all words $u, v$ and all letters $a, b$ by

$$
u \sqcup 1=1 \sqcup u=u, \quad u a \sqcup v b=(u a \sqcup v) b+(u \sqcup v b) a .
$$

Remark 5. To distinguish between the free Hopf algebra and its graded dual, we denote them by $\left(\mathbb{k} A^{*}, ., \Delta\right)$ and $\left(\mathbb{k} A^{*}, \omega, \delta\right)$ respectively, skipping the unit and counit symbols.

\subsection{Hopf algebra structures on rooted ordered coloured trees}

Below we present (slightly reformulated — see the first Remark after Proposition 6) results by Foissy [29] relevant to our paper.

A rooted ordered tree (called also rooted plane tree) is a finite rooted tree $t$ such that for each vertex $v$ of $t$, the children of $v$ are totally ordered (from left to right on our pictures). Together with the depth partial order (defined by the distance from the root) this induces linear order on the vertex set $V(t)$ of the tree obtained from left-toright depth-first search; see Figure 1. By the trivial rooted tree we understand the tree 


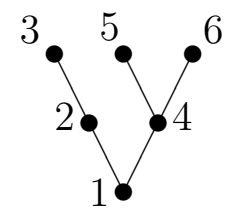

Figure 1: A rooted ordered tree with induced natural linear order on the vertex set

consisting of the root only. A planted rooted tree is a non-trivial rooted tree such that its root has only one child.

A rooted ordered coloured (ROC) tree is a rooted ordered tree $t$ together with a function from a set $E(t)$ of its edges to the set $A$ of colours, we assume $|A|<\infty$. By $\mathbb{k} T_{A}$ denote the linear space of finite formal combinations of A-coloured rooted ordered trees with coefficients in the field $\mathbb{k}$. The space $\mathbb{k} T_{A}$ is graded

$$
\mathbb{k} T_{A}=\oplus_{k \geqslant 0} \mathbb{k} T_{A}^{(k)}, \quad T_{A}^{(k)}=\{t:|t|=k\},
$$

with the weight $|t|$ of a ROC-tree $t$ being the number of its edges.

By the well known connection [72] between rooted ordered trees and Catalan numbers $C_{k}$, dimension of each graded component $\mathbb{k} T_{A}^{(k)}$ equals

$$
\operatorname{dim} \mathbb{k} T_{A}^{(k)}=|A|^{k} C_{k}=\frac{|A|^{k}}{k+1}\left(\begin{array}{c}
2 k \\
k
\end{array}\right) .
$$

Define the product "." on $\mathbb{k} T_{A}$ as the concatenation of trees by identification of their roots; see Figure 2 for an example. The product respects the gradation, is associative

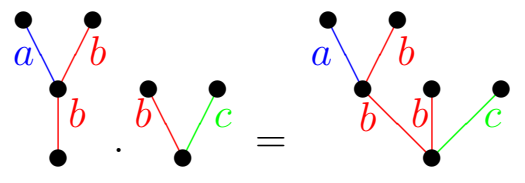

Figure 2: Multiplication of two coloured ordered rooted trees

with the trivial tree $\bullet$ being the neutral element (i.e. the unit map $\eta: \mathbb{k} \rightarrow \mathbb{k} T_{A}$ is defined by $1 \longmapsto \bullet$ ).
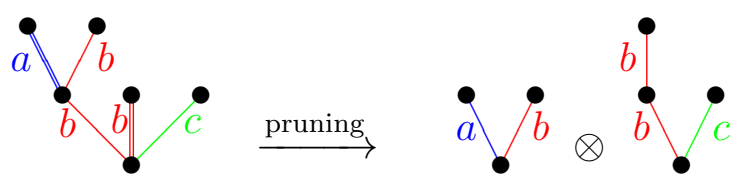

Figure 3: Pruning of a ROC-tree; pruned branches are thickened

In order to define compatible coproduct on $\mathbb{k} T_{A}$ one has first to describe the operation of pruning of a tree. A rooted subtree $t_{s}$ of a ROC-tree $t$ is called admissible if it shares 
the root of $t$. Such an admissible subtree is again ROC-tree with the root, order and colours inherited from $t$. The set of admissible subtrees of $t$ (including the trivial tree and $t$ itself) will be denoted by $A(t)$. Given such admissible subtree $t_{s} \subset t$, the planted trees being branches of $t$ pruned to get $t_{s}$, ordered according to the linear order on $t$ give the sequence $\left(t_{1}, \ldots, t_{m}\right)$. By concatenation of the sequence of pruned branches we obtain the complementary tree $t_{c}=t_{1} \cdot \ldots \cdot t_{m}$ to the admissible subtree $t_{s}$ of $t$. Such a pruning operation gives an element $t_{c} \otimes t_{s}$; see Figure 3 for an example.

The coproduct of a tree is defined as sum of pairs $t_{c} \otimes t_{s}$ for all admissible subtrees of $t$; see Figure 4

$$
\Delta(t)=\sum_{t_{s} \in A(t)} t_{c} \otimes t_{s}
$$

and then extended to $\mathbb{k} T_{A}$ by linearity.

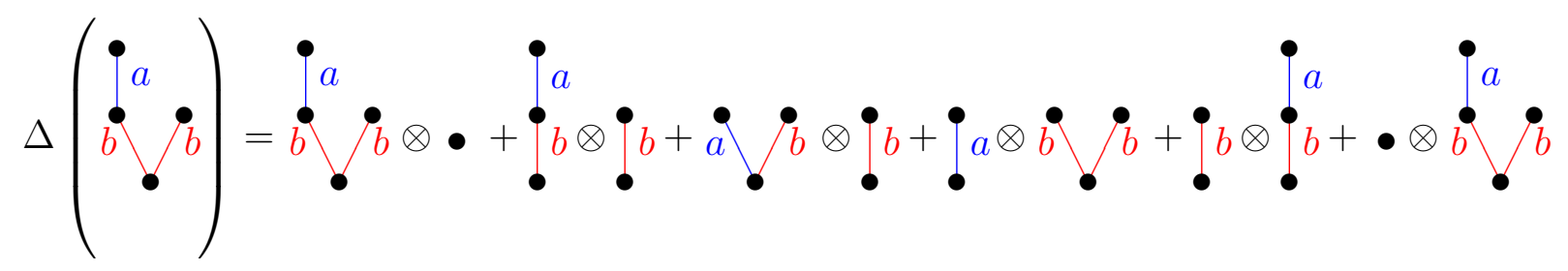

Figure 4: The pruning coproduct of a ROC-tree

Such coproduct is coassociative, respects the gradation, and is compatible with the counit defined on trees as

$$
\epsilon(t)= \begin{cases}1 & \text { if } t=\bullet \\ 0 & \text { otherwise }\end{cases}
$$

In this context it is convenient to define the operation $B_{i}^{+}$of planting of a tree on a new root by attaching it to the old one by additional edge coloured by $i$. In particular, planting allows to define the coproduct recursively starting from $\Delta(\bullet)=\bullet \otimes \bullet$, and using then the formula

$$
\Delta\left(B_{i}^{+}(t)\right)=B_{i}^{+}(t) \otimes \bullet+\left(\mathrm{id} \otimes B_{i}^{+}\right) \circ \Delta(t),
$$

together with compatibility of the coproduct $\Delta$ with the concatenation product. Equation (2.19) has the following simple meaning: apart from the trivial subtree, all admissible subtrees of a planted tree contain the lowest (i.e. incident to the root) edge.

Proposition 6. The concatenation multiplication and pruning coproduct with the corresponding unit and counit maps equip $\mathbb{k} T_{A}$ with the structure of graded locally finite and connected bialgebra (thus Hopf algebra).

Remark 7. The above result was given by Foissy [29] in the equivalent setting of the rooted ordered vertex-coloured (or decorated) forests. Any ROC tree is uniquely mapped, by deletion of the root, to an ordered forest colouring first its vertices using colours of adjacent 
edges below them; see the bijection map visualized on Figure 5. This notation resulted as decorated and non-commutative version of the Connes-Kreimer Hopf algebra [13] used to explain the renormalization procedure in the quantum field theory.

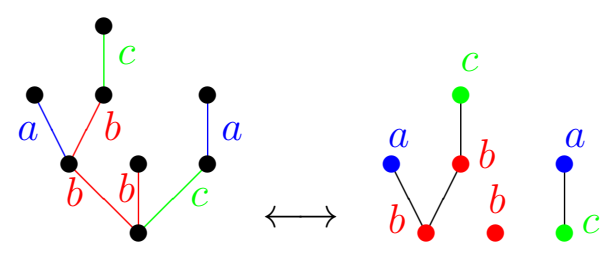

Figure 5: Transition from the setting of ROC-trees to the setting of ROD-forests

Remark 8. In [29] one can find, among others, also the corresponding description of the antipode, which can be transferred from the ROD-forests to ROC-trees.

Corollary 9. The subalgebra of $\mathbb{k} T_{A}$ generated by one-edge planted trees $a_{i}=B_{i}^{+}(\bullet)$ is a Hopf subalgebra isomorphic to the free Hopf algebra described in Example 1.

We conclude this Section by presenting the graded dual of the Hopf algebra of ROCtrees, which is again reformulation of the corresponding results of [29]. Because the natural basis of the finite dimensional subspace $\mathbb{k} T_{A}^{(k)}$ is provided by ROC-trees of weight $k$ it seems natural to represent the dual basis of $\left(\mathbb{k} T_{A}^{(k)}\right)^{*}$ by such trees again, i.e. the functional $\phi_{t} \in\left(\mathbb{k} T_{A}^{(k)}\right)^{*}$ defined on trees by

$$
\left\langle\phi_{t}, t^{\prime}\right\rangle= \begin{cases}1 & \text { if } t=t^{\prime} \\ 0 & \text { otherwise }\end{cases}
$$

by standard abuse of notation is identified with $t$. The dual (deconcatenation) coproduct $\delta=(.)^{*}$ to the concatenation product acts on trees as

$$
\delta(t)=\sum_{t^{\prime}, t^{\prime \prime} \in T_{n}}\left\langle t, t^{\prime} \cdot t^{\prime \prime}\right\rangle t^{\prime} \otimes t^{\prime \prime}=\sum_{t^{\prime} \cdot t^{\prime \prime}=t} t^{\prime} \otimes t^{\prime \prime},
$$

i.e. when $t=t_{1} \ldots t_{m}$ is planted trees decomposition, then

$$
\delta\left(t_{1} \ldots t_{m}\right)=\sum_{i=0}^{m}\left(t_{1} \ldots t_{i}\right) \otimes\left(t_{i+1} \ldots t_{m}\right),
$$

see Figure 6.

Corollary 10. Equation (2.21) implies the following matching condition between the deconcatenation coproduct and the concatenation product

$$
\delta(s . t)=\delta(s) \cdot(\bullet \otimes t)+(s \otimes \bullet) . \delta(t)-s \otimes t, \quad s, t \in T_{A},
$$

where, by the standard abuse of notation, we extended the product sign from $\mathbb{k} T_{A}$ to $\mathbb{k} T_{A} \otimes$ $\mathbb{k} T_{A}$. 


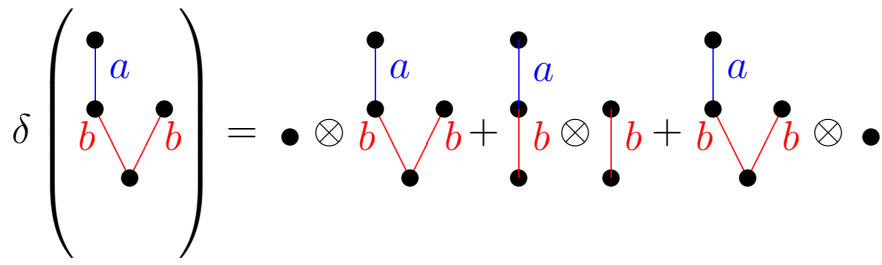

Figure 6: The deconcatenation coproduct of a ROC-tree

The (asymmetric shuffle or grafting) product $\stackrel{T}{\amalg}=\Delta^{*}$, dual to the pruning coproduct satisfies

$$
\Delta(t)=\sum_{t^{\prime}, t^{\prime \prime} \in T_{A}}\left\langle t, t^{\prime} \stackrel{T}{\amalg} t^{\prime \prime}\right\rangle t^{\prime} \otimes t^{\prime \prime},
$$

and is defined with the help of the grafting procedure that follows from comparison of equations (2.17) and (2.24). Given ROC-tree $t^{\prime}=t_{1} \ldots, t_{m}$ decomposed into the planted factors, its (non-unique) grafting on ROC-tree $t^{\prime \prime}$ is defined as attaching roots of the factors $t_{i}$ to vertices of $t^{\prime \prime}$ in a way, which preserves the original ordering of the factors. In other words, a grafting of $t^{\prime}$ on $t^{\prime \prime}$ gives a tree $\tilde{t}$ such that there exists a pruning with $t^{\prime \prime}=\tilde{t}_{s}$ with the corresponding $t^{\prime}=\tilde{t}_{c}$; see Figure 7 for an example.

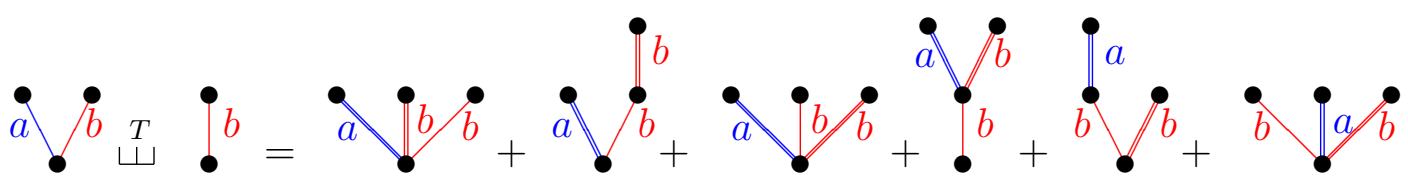

Figure 7: The asymmetric shuffle (or grafting) product of two ROC-trees; grafted branches are thickened

With the grafting product $\stackrel{T}{\uplus}$, deconcatenation coproduct $\delta$, the unit $\eta=\epsilon^{*}$ and counit $\epsilon=\eta^{*}$ maps, the space spanned by ROC-trees is equipped with another bialgebra (thus Hopf algebra) structure - the graded dual to the previous one.

Remark 11. It is remarkable fact, discovered by Foissy [29], that the duality described above is self-duality. The situation is analogous to the well known self-duality of the Hopf algebra of symmetric functions [72].

\subsection{Hopf algebra of quasi-symmetric functions, and its graded dual}

Let $x=\left(x_{1}, x_{2}, x_{3}, \ldots\right)$ denote infinite totally ordered set of commuting variables, and let $\mathbb{k}\left[\left[x_{1}, x_{2}, x_{3}, \ldots\right]\right]$ be the algebra of formal power series of bounded degree. Such a formal series is called quasi-symmetric function if the coefficient of any term $x_{i_{1}}^{n_{1}} x_{i_{2}}^{n_{2}} \ldots x_{i_{k}}^{n_{k}}$ with $i_{1}<i_{2}<\cdots<i_{k}$ strictly increasing, agrees with that of $x_{1}^{n_{1}} x_{2}^{n_{2}} \ldots x_{k}^{n_{k}}$. The linear space QSym of quasi-symmetric functions has as a basis the monomial quasi-symmetric 
functions indexed by compositions. Recall that a composition of $m$, written $\alpha \models m$, is a finite sequences of positive integers $\alpha=\left(\alpha_{1}, \alpha_{2}, \ldots, \alpha_{k}\right)$ such that $|\alpha|=\alpha_{1}+\alpha_{2}+\cdots+\alpha_{k}=$ $m$. In this case we say that $\alpha$ has $k$ parts, or it is of length $\ell(\alpha)=k$. The elements of the basis are of the form

$$
M_{\alpha}=\sum_{i_{1}<i_{2}<\cdots<i_{k}} x_{i_{1}}^{\alpha_{1}} x_{i_{2}}^{\alpha_{2}} \ldots x_{i_{k}}^{\alpha_{k}}
$$

where the sum is over all $k$-tuples $\left(i_{1}, i_{2}, \ldots, i_{k}\right)$ of strictly increasing; by definition $M_{\emptyset}=1$. The algebra QSym is graded with each graded component QSym $^{(m)}$ spanned by those $M_{\alpha}$ for which $|\alpha|=m$. By the well known bijection [51] any such composition can be identified with a subset

$$
\operatorname{set}(\alpha)=\left\{\alpha_{1}, \alpha_{1}+\alpha_{2}, \ldots, \alpha_{1}+\cdots+\alpha_{k-1}\right\}
$$

of $\{1,2, \ldots, m-1\}$, therefore $\operatorname{dim} \mathrm{QSym}^{(m)}=2^{m-1}$.

We can put a partial order on the set of all compositions of $m$ by refinement. The covering relations are of the form

$$
\left(\alpha_{1}, \ldots, \alpha_{i}, \alpha_{i+1}, \ldots \alpha_{k}\right) \prec\left(\alpha_{1}, \ldots, \alpha_{i}+\alpha_{i+1}, \ldots \alpha_{k}\right) .
$$

This allows to define another important basis formed by the fundamental quasi-symmetric functions, also indexed by compositions

$$
F_{\alpha}=\sum_{\beta \preccurlyeq \alpha} M_{\beta} .
$$

By inclusion-exclusion we can express the $M_{\alpha}$ in terms of the $F_{\alpha}$

$$
M_{\alpha}=\sum_{\beta \preccurlyeq \alpha}(-1)^{\ell(\beta)-\ell(\alpha)} F_{\beta} .
$$

The product in QSym, inherited from the standard multiplication of power series, can be described in the basis $\left(M_{\alpha}\right)$ in terms of the quasi-shuffle (or overlapping shuffle) $\stackrel{Q}{\sqcup}$ of compositions: in addition to shuffling components $\alpha_{i}$ and $\beta_{j}$ of two compositions $\alpha=\left(\alpha_{1}, \ldots, \alpha_{k}\right)$ and $\beta=\left(\beta_{1}, \ldots, \beta_{l}\right)$ we may replace any number of pairs of consecutive components $\alpha_{i}$ and $\beta_{j}$ in the shuffle by their sum $\alpha_{i}+\beta_{j}$

$$
M_{\alpha} M_{\beta}=\sum_{\gamma} M_{\gamma}
$$

where $\gamma$ is a summand in quasi-shuffle of $\alpha$ and $\beta$.

Example 12. For $M_{(1)}=x_{1}+x_{2}+\cdots$ and $M_{(2)}=x_{1}^{2}+x_{2}^{2}+\cdots$ we have

$\left(x_{1}+x_{2}+\cdots\right)\left(x_{1}^{2}+x_{2}^{2}+\cdots\right)=\left(x_{1} x_{2}^{2}+x_{1} x_{3}^{2}+\cdots\right)+\left(x_{1}^{2} x_{2}+x_{1}^{2} x_{3}+\cdots\right)+\left(x_{1}^{3}+x_{2}^{3}+\cdots\right)$,

therefore we obtain

$$
M_{(1)} M_{(2)}=M_{(1,2)}+M_{(2,1)}+M_{(3)}, \quad \text { or } \quad(1) \stackrel{Q}{\amalg}(2)=(1,2)+(2,1)+(3) .
$$


The coproduct $\delta$ in the algebra of quasi-symmetric functions can be defined using the doubling variables trick. Here to the totally ordered set of variables $x=\left(x_{1}, x_{2}, x_{3}, \ldots\right)$ we add its copy $y=\left(y_{1}, y_{2}, y_{3}, \ldots\right)$ placing elements of $y$ after elements of $x$, and getting the ordered sum of the sets of variables. To obtain the coproduct $\delta(f)$ of a quasi-symmetric function $f$ we expand the function over the doubled variables, decompose resulting expression into sum of products of functions of $x$ and $y$ getting this way

$$
f \mapsto f(x) \mapsto f(x, y)=\sum_{j} f_{j}^{\prime}(x) f_{j}^{\prime \prime}(y) \mapsto \sum_{j} f_{j}^{\prime} \otimes f_{j}^{\prime \prime}=\delta(f) .
$$

In the basis of monomial quasi-symmetric functions $\left(M_{\alpha}\right)$ the coproduct formula reads

$$
\delta\left(M_{\alpha}\right)=\sum_{\beta \cdot \gamma=\alpha} M_{\beta} \otimes M_{\gamma}
$$

where $\beta \cdot \gamma$ is concatenation of two compositions. As a result we obtain graded, locally finite and connected bialgebra (thus Hopf algebra) which is commutative but not cocommutative.

Example 13. Applying the procedure to $M_{(2,1)}=x_{1}^{2} x_{2}+x_{1}^{2} x_{3}+\cdots$ we have

$$
\begin{aligned}
x_{1}^{2} x_{2}+x_{1}^{2} x_{3}+\cdots & \mapsto x_{1}^{2} x_{2}+x_{1}^{2} x_{3}+\cdots+x_{1}^{2} y_{1}+x_{1}^{2} y_{2}+\cdots+y_{1}^{2} y_{2}+y_{1}^{2} y_{3}+\cdots= \\
& =M_{(2,1)}(x)+M_{(2)}(x) M_{(1)}(y)+M_{(2,1)}(y)
\end{aligned}
$$

getting this way

$$
\delta\left(M_{(2,1)}\right)=M_{(2,1)} \otimes 1+M_{(2)} \otimes M_{(1)}+1 \otimes M_{(2,1)} .
$$

The graded dual to QSym is called the Hopf algebra of non-commutative symmetric functions [32] and denoted by NSym. Let $\left(H_{\alpha}\right)$ be the dual basis to $\left(M_{\beta}\right)$

$$
\left\langle H_{\alpha}, M_{\beta}\right\rangle=\delta_{\alpha \beta},
$$

then by dualization of equations (2.30) and (2.33) we obtain the product and coproduct formulas in NSym

$$
H_{\alpha} H_{\beta}=H_{\alpha \cdot \beta}, \quad \Delta\left(H_{\alpha}\right)=\sum_{(\beta, \gamma)} H_{\beta} \otimes H_{\gamma},
$$

where $\alpha$ can be obtained as a summand in quasi-shuffle of $\beta$ and $\gamma$. In particular, for a composition $\alpha=\left(\alpha_{1}, \ldots, \alpha_{k}\right)$ one has

$$
H_{\alpha}=H_{\alpha_{1}} \ldots H_{\alpha_{k}}
$$

where we wrote $H_{j}=H_{(j)}$ for a composition $(j)$. The dual element to $M_{\emptyset}=1$ is $H_{\emptyset}=H_{0}=1$. This leads to the conclusion that, as algebra, NSym is freely generated by non-commuting elements $H_{1}, H_{2}, \ldots$

$$
\mathrm{NSym}=\mathbb{k}\left\langle H_{1}, H_{2}, \ldots\right\rangle .
$$


The coproduct formula for the generators follows from equations (2.36) and reads

$$
\Delta\left(H_{m}\right)=\sum_{j=0}^{m} H_{j} \otimes H_{m-j} .
$$

Remark 14. It is known [39] that the Hopf algebra NSym is isomorphic to a Hopf subalgebra of rooted ordered (monochromatic) trees generated by $\left(B^{+}\right)^{m}(\bullet) \leftrightarrow H_{m}$, where in the monochromatic $|A|=1$ case we skip the lower index $i=1$ describing the colour of the attached edge.

Remark 15. One can recapitulate this Section in the spirit of Examples 1 and 3 that we presented two, mutually dual, Hopf algebra structures in the space of compositions. Similarly to the shuffle product of words, the quasi-shuffle of compositions can be defined recursively [38] for all compositions $\alpha, \beta$ and all natural numbers $k, l$ by

$$
\begin{gathered}
\alpha \stackrel{Q}{\amalg} \emptyset=\emptyset \stackrel{Q}{\sqcup} \alpha=\alpha, \\
((k) \cdot \alpha) \stackrel{Q}{\amalg}((l) \cdot \beta)=(k) \cdot(\alpha \stackrel{Q}{\sqcup}((l) \cdot \beta))+(l) \cdot(((k) \cdot \alpha) \stackrel{Q}{\sqcup} \beta)+(k+l) \cdot(\alpha \stackrel{Q}{\sqcup} \beta) .
\end{gathered}
$$

\section{The Hopf algebra of coloured non-commutative symmetric functions}

\subsection{The Hopf algebra of sentences}

Given finite alphabet $A=\left\{a_{1}, \ldots, a_{n}\right\}$, recall (see Example 1) that words over $A$ are finite sequences of its elements (letters) written without separation. Define sentences as finite sequences of words (instead of spacing to separate them we use commas). The size of a word $w=a_{i_{1}} \ldots a_{i_{k}}$ is the number $|w|=k$ of its letters, the size of a sentence $I=\left(w_{1}, w_{2}, \ldots, w_{m}\right)$ is the sum $|I|=\left|w_{1}\right|+\left|w_{2}\right|+\cdots+\left|w_{m}\right|$ of sizes of its words, while the length of the sentence is the number $\ell(I)=m$ of its words. The maximal word of the sentence is the concatenation $w(I)=w_{1} w_{2} \ldots w_{m}$ of all its words.

Example 16. The sentence $I=(a b a, c a, b a c)$ over alphabet $\{a, b, c\}$ has length $\ell(I)=3$, is of size $|I|=8$. Its maximal word is $w(I)=$ abacabac.

Remark 17. For unary alphabet $A=\{a\}$ we will identify words with their size, and then identify sentences with corresponding compositions, for example $(a a a, a a, a a a) \longleftrightarrow$ $(3,2,3)$. Speaking about colours in the place of letters, instead of sentences we may use the notion of coloured compositions.

Given two sentences $I, J$, we say that $I$ is coarsening of $J$ (or equivalently, $J$ is refinement of $I$ ), denoted by $I \succcurlyeq J$, if we can obtain the words of $I$ by concatenation of adjacent words of $J$. For example $(a b a, c a, b a c) \succcurlyeq(a b, a, c a, b a, c)$. With the refinement order, the poset of sentences having the same maximal word $w$ is isomorphic to the poset of compositions of $|w|$, and therefore isomorphic (recall the bijection set mentioned in Section 2.3) to the (dual of the) Boolean poset of $\{1,2, \ldots,|w|-1\}$. This allows to 

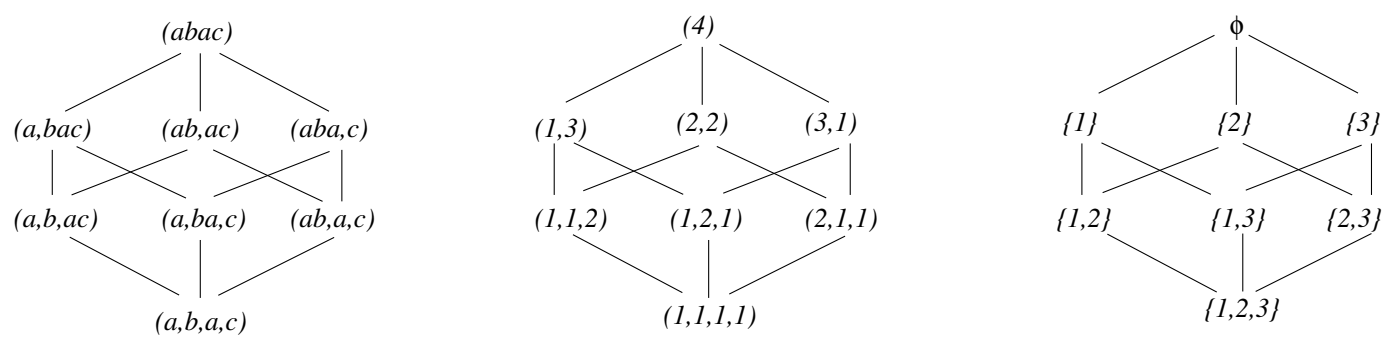

Figure 8: Isomorphism of three posets

transfer structural results between the posets, in particular to find description of the Moebius function of the posets of sentences

$$
\mu(J, I)=(-1)^{\ell(J)-\ell(I)} \quad \text { for } \quad J \preccurlyeq I .
$$

Remark 18. The above poset point of view makes the theory of coloured non-commutative symmetric and quasi-symmetric functions so similar to the original monochromatic theory. There are however various differences related to new degree of non-commutativity caused by presence of different colours/letters.

Corollary 19. The set of sentences of size $n \in \mathbb{N}$ splits onto $|A|^{n}$ pairwise disjoint classes of sentences with the same maximal word.

Define also two involutions acting on sentences: reversal and complement. The reversal of $I$, denoted by $I^{r}$ is obtained by writing the words of $I$ in the reverse order

$$
\left(w_{1}, w_{2}, \ldots, w_{m}\right)^{r}=\left(w_{m}, w_{m-1}, \ldots, w_{1}\right) .
$$

The complement of $I$, denoted by $I^{c}$ is the sentence with the same maximal word as $I$ but whose image under the map set is the complementary subset of $\{1,2, \ldots,|I|-1\}$. Equivalently, in the maximal word $w(I)$ we put separating commas between letters if there was no comma between the letters in $I$.

Example 20. For $I=(a b a, c a, b a c)$ we have $I^{r}=(b a c, c a, a b a)$ and $I^{c}=(a, b, a c, a b, a, c)$

We may represent sentences in terms of ribbons placing its words in subsequent rows such that the first letter of the next word is exactly below the last letter of the previous one. Then the ribbon diagram of $I^{c}$ is obtained by transposition of the ribbon diagram of $I$, see Figure 9 .

Remark 21. From yet another point of view, we may identify words with the so called planted tall trees (or ladders)

$$
w=a_{i_{1}} a_{i_{2}} \ldots a_{i_{k}} \longleftrightarrow\left(B_{i_{k}}^{+} \circ \cdots \circ B_{i_{1}}^{+}\right)(\bullet) .
$$

Then sentences are in correspondence with concatenations of such trees, and are called tall trees; see Figure 10 for an example. 


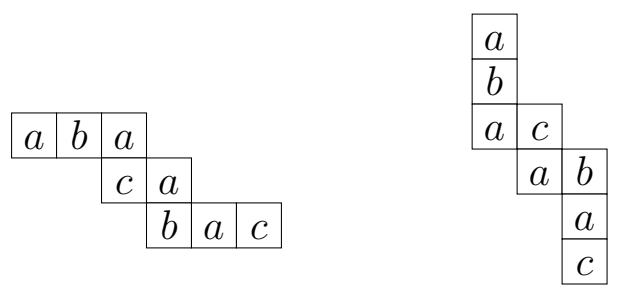

Figure 9: The ribbon diagrams of the sentence $(a b a, c a, b a c)$ and of its complement

Corollary 22. The set of sentences over A is bijective with the context-free language [73] generated by grammar with

- terminal symbols $A \cup \bar{A}$, where $\bar{A}$ is the disjoint copy of $A$ with elements $\bar{a}$ for $a \in A$,

- nonterminal symbols $\{X, Z\}$ with $X$ being the initial symbol of the grammar,

- production rules

$$
X \rightarrow 1\left|X Z, \quad Z \rightarrow \bar{a}_{i} Z a_{i}\right| \bar{a}_{i} a_{i}, \quad \text { where } a_{i} \in A .
$$

Roughly speaking, the second rule produces words, while the first rule builds sentences from words. The relation of the Hopf algebra of trees and its subalgebras to context-free languages will be presented in another publication.

Example 23. The element of the context-free language described above, which corresponds to the sentence $(a b a, c a, b a c)$ is $\bar{a} \bar{b} \bar{a} a b a \bar{a} \bar{c} c a \bar{c} \bar{a} \bar{b} b a c$.

Given two sentences $I=\left(w_{1}, \ldots, w_{m}\right), J=\left(v_{1}, \ldots, v_{n}\right)$ their concatenation $I \cdot J$ is the sentence $\left(w_{1}, \ldots, w_{m}, v_{1}, \ldots, v_{n}\right)$ obtained by juxtaposition of the sequences of their words, and corresponds to joining bunches of tall trees. Their near-concatenation $I \odot J$ is the sentence $\left(w_{1}, \ldots, w_{m} v_{1}, \ldots, v_{n}\right)$ in which the last word of $I$ is concatenated with the first word of $J$ For example

$$
(a b a, c b) \cdot(b b, a c)=(a b a, c b, b b, a c), \quad(a b a, c b) \odot(b b, a c)=(a b a, c b b b, a c) .
$$

Notice that since an admissible subtree of a tall tree and its complementary tree are also of such form then the pruning coproduct (2.17) of tall trees doesn't lead out of that space.

Proposition 24. The planted tall trees generate Hopf subalgebra of ROC-trees with the concatenation multiplication and pruning coproduct.

To describe the corresponding comultiplication in the language of sentences (coloured compositions) let us consider also weak sentences, which may contain empty words. Given weak sentence $I$, by $\widetilde{I}$ we denote the corresponding sentence obtained by removing the empty words from it (or the empty word if $I$ consists of the empty words only). We say that the weak sentence $J=\left(v_{1}, \ldots, v_{m}\right)$ is contained in the sentence $I=\left(w_{1}, \ldots, w_{m}\right)$, denoted by $J \subset I$, if there exists complementary weak sentence $\left(u_{1}, \ldots, u_{m}\right)$, denoted by $I \backslash J$, such that $w_{i}=u_{i} v_{i}$ for $i=1, \ldots, m$. 


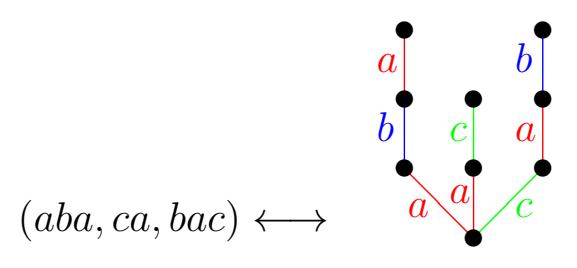

Figure 10: Sentences as coloured tall trees

Remark 25. To avoid confusion we recall that the empty sentence, in accordance to our previous notation, is denoted by by 1 , the corresponding empty composition was denoted previously by $\emptyset$, and the corresponding trivial tree by

Proposition 26. The algebra of sentences (coloured compositions) is a bialgebra with the multiplication being concatenation of sentences, the comultiplication given by

$$
\Delta(I)=\sum_{J \subset I} \widetilde{I \backslash J} \otimes \widetilde{J}
$$

the natural unity map, and the counit

$$
\epsilon(I)= \begin{cases}1 & \text { if } I=1 \\ 0 & \text { otherwise }\end{cases}
$$

Example 27. The coproduct of a ROC-tree visualized in Figure 4 reads in the present setting as follows

$$
\begin{aligned}
\Delta(a b, b) & =(a b, b) \otimes 1+(a b) \otimes(b)+(a, b) \otimes(b)+(a) \otimes(b, b)+(b) \otimes(a b)+1 \otimes(a b, b)= \\
& =((a b) \otimes 1+(a) \otimes(b)+1 \otimes(a b)) \cdot((b) \otimes 1+1 \otimes(b)) .
\end{aligned}
$$

Remark 28. As the above Example shows, the comultiplication in the Hopf algebra of sentences is not cocommutative.

Corollary 29. The bialgebra of sentences over alphabet $A$ is graded, with the weight of a sentence being its size, locally finite and connected (thus Hopf algebra). The dimension of the graded component consisting of sentences of size $m>0$ is $|A|^{m} 2^{m-1}$.

Corollary 30. The action of the comultiplication on single-word generators reads as follows

$$
\Delta\left(a_{i_{1}} a_{i_{2}} \ldots a_{i_{k}}\right)=\sum_{j=0}^{k}\left(a_{i_{1}} a_{i_{2}} \ldots a_{i_{j}}\right) \otimes\left(a_{i_{j+1}} \ldots a_{i_{k}}\right) .
$$

Remark 31. In the present setting the free Hopf algebra of Example 1 should be identified with the subalgebra of sentences built out of single-letter words, see also Corollary 9. 
In [29] one can find also detailed description of the antipode of the Hopf algebra of ROD-forests, which can be used to define the antipode of the Hopf algebra of tall trees, and thus to transfer it into the language of the Hopf algebra of sentences. To make the paper self-contained we perform below the corresponding calculation from scratch avoiding this route.

Proposition 32. The antipode in the Hopf algebra of sentences is given by the following formula

$$
S(I)=\sum_{J \preccurlyeq I^{r}}(-1)^{\ell(J)} J .
$$

Proof. We will show first that the above formula gives the antipode for single-word sentences, which generate the algebra of sentences. The coproduct formula (3.9) and equation (2.6) give the recurrence relation

$$
S\left(a_{i_{1}} a_{i_{2}} \ldots a_{i_{k}}\right)=-\sum_{j=0}^{k-1} S\left(a_{i_{1}} a_{i_{2}} \ldots a_{i_{j}}\right) \cdot\left(a_{i_{j+1}} \ldots a_{i_{k}}\right),
$$

which, in particular, for $k=1$ gives the correct formula

$$
S\left(a_{i}\right)=-\left(a_{i}\right) .
$$

Assume that the expression for the antipode holds true for generators indexed by single-word sentences of size not greater than $k$, then for $k+1$ we have

$$
S\left(a_{i_{1}} a_{i_{2}} \ldots a_{i_{k+1}}\right)=\sum_{j=0}^{k}\left(\sum_{J \preccurlyeq\left(a_{i_{1}} a_{i_{2}} \ldots a_{i_{j}}\right)}(-1)^{\ell(J)+1} J \cdot\left(a_{i_{j+1}} \ldots a_{i_{k+1}}\right)\right) .
$$

which gives the correct expression, because we separated the last word of the sentence refining $\left(a_{i_{1}} a_{i_{2}} \ldots a_{i_{k+1}}\right)$.

By the anti-endomorphism property of the antipode we have

$$
\begin{aligned}
S\left(w_{1}, \ldots, w_{m}\right) & =S\left(w_{m}\right) \cdot \ldots \cdot S\left(w_{1}\right)=\sum_{J_{m} \preccurlyeq\left(w_{m}\right), \ldots J_{1} \preccurlyeq\left(w_{1}\right)}(-1)^{\ell\left(J_{m}\right)+\cdots+\ell\left(J_{1}\right)} J_{m} \cdot \ldots \cdot J_{1}= \\
& =\sum_{J_{m} \ldots J_{1} \preccurlyeq\left(w_{m}, \ldots, w_{1}\right)}(-1)^{\ell\left(J_{m}\right)+\cdots+\ell\left(J_{1}\right)} J_{m} \cdot \ldots \cdot J_{1}=\sum_{J \preccurlyeq\left(w_{1}, \ldots, w_{m}\right)^{r}}(-1)^{\ell(J)} J,
\end{aligned}
$$

what concludes the proof.

Remark 33. In $[2,5,42,57,66]$ another notion of coloured compositions is considered. In our approach such a variant corresponds to sentences made of words with definite colours, for example $(b b b, a, b b, c c c c)$. Because concatenation and pruning operations leave such property untouched one obtains this way a Hopf subalgebra of that introduced in this Section. Notice [42] that the dimension of the graded component consisting of such compositions/sentences of size $m>0$ is $|A|(|A|+1)^{m-1}$, to be compared with the dimension calculated in Corollary 29. 


\subsection{Coloured non-commutative symmetric functions}

Because of the isomorphism of the Hopf algebra of sentences on unary alphabet with the Hopf algebra NSym of non-commutative symmetric functions, the algebra of sentences over alphabet $A$ can be also called the algebra of coloured non-commutative symmetric functions, and denoted by $\mathrm{NSym}_{A}$. We will discuss also other bases of $\mathrm{NSym}_{A}$ indexed by sentences, therefore the linear basis of sentences will be denoted from now on by $\left(H_{I}\right)$ and called the basis of complete homogeneous coloured non-commutative symmetric functions. The multiplication, comultiplication and the antipode in the new notation read

$$
\begin{aligned}
H_{I} \cdot H_{J} & =H_{I \cdot J}, \\
\Delta\left(H_{I}\right) & =\sum_{J \subset I} H_{\widetilde{I \backslash J}} \otimes H_{\widetilde{J}}, \\
S\left(H_{I}\right) & =\sum_{J \preccurlyeq I^{r}}(-1)^{\ell(J)} H_{J} .
\end{aligned}
$$

In particular, for functions indexed by single-word sentences we have

$$
\Delta\left(H_{\left(a_{i_{1}} a_{i_{2}} \ldots a_{i_{k}}\right)}\right)=\sum_{j=0}^{k} H_{\left(a_{i_{1}} a_{i_{2}} \ldots a_{i_{j}}\right)} \otimes H_{\left(a_{i_{j+1}} \ldots a_{i_{k}}\right)},
$$

and the recursive formula (2.6) then reads

$$
S\left(H_{\left(a_{i_{1}} a_{i_{2}} \ldots a_{i_{k}}\right)}\right)=\left\{\begin{array}{l}
\left.-\sum_{j=0}^{k-1} S\left(H_{\left(a_{i_{1}} a_{i_{2}} \ldots a_{i_{j}}\right)}\right) \cdot H_{\left(a_{i_{j+1}} \ldots a_{i_{k}}\right)}\right), \\
-\sum_{j=1}^{k} H_{\left(a_{i_{1}} a_{i_{2}} \ldots a_{i_{j}}\right)} \cdot S\left(H_{\left(a_{i_{j+1}} \ldots a_{i_{k}}\right)}\right) .
\end{array}\right.
$$

Remark 34. We define the operations of reversal and complement in the basis $\left(H_{I}\right)$

$$
r\left(H_{I}\right)=H_{I^{r}}, \quad c\left(H_{I}\right)=H_{I^{c}}
$$

and extend them to $\mathrm{NSym}_{A}$ by linearity.

\section{Example 35.}

$$
S\left(H_{(a b, c)}\right)=S\left(H_{(c)}\right) \cdot S\left(H_{(a b)}\right)=\left(-H_{(c)}\right) \cdot\left(-H_{(a b)}+H_{(a, b)}\right)=H_{(c, a b)}-H_{(c, a, b)} .
$$

Like in the classical case define coloured non-commutative elementary symmetric functions by

$$
E_{I}=\sum_{J \preccurlyeq I}(-1)^{|I|-\ell(J)} H_{J},
$$

which allows to rewrite the antipode of the complete homogeneous functions as

$$
S\left(H_{I}\right)=(-1)^{|I|} E_{I^{r}} .
$$

Remark 36. In general $E_{I^{r}} \neq r\left(E_{I}\right)$, for example

$$
E_{(a b, c)^{r}}=H_{(c, a, b)}-H_{(c, a b)}, \quad \text { while } \quad r\left(E_{(a b, c)}\right)=H_{(c, b, a)}-H_{(c, a b)} .
$$


Proposition 37. The product of coloured non-commutative elementary symmetric functions satisfies the formula

$$
E_{I} \cdot E_{J}=E_{I \cdot J}
$$

in particular, the elementary symmetric functions are generated by single-word elementary functions.

Proof. By equation (3.23) and anti-endomorphism property of the antipode we have

$$
E_{I} \cdot E_{J}=(-1)^{|I|+|J|} S\left(H_{I^{r}}\right) \cdot S\left(H_{J^{r}}\right)=(-1)^{|I \cdot J|} S\left(H_{J^{r} \cdot I^{r}}\right)=(-1)^{|I \cdot J|} S\left(H_{(I \cdot J)^{r}}\right)=E_{I \cdot J} .
$$

Proposition 38. The coloured non-commutative elementary symmetric functions form a linear basis of the Hopf algebra $\mathrm{NSym}_{A}$, in particular

$$
H_{I}=\sum_{J \preccurlyeq I}(-1)^{|I|-\ell(J)} E_{J} .
$$

Proof. The right hand side of equation (3.26) reads

$$
\sum_{J \preccurlyeq I}(-1)^{|I|-\ell(J)} \sum_{K \preccurlyeq J}(-1)^{|J|-\ell(K)} H_{K}=\sum_{K \preccurlyeq I}(-1)^{\ell(I)-\ell(K)} H_{K} \sum_{K \preccurlyeq J \preccurlyeq I}(-1)^{\ell(I)-\ell(J)},
$$

where we changed the order of summation using also the fact that $|I|=|J|$. By properties of the Moebius function (3.1) the second sum equals 1 for $K=I$ and vanishes otherwise, what concludes the proof.

Corollary 39. The single-word coloured non-commutative elementary symmetric functions generate the algebra $\mathrm{NSym}_{A}$.

Because for $|A|>1$ the Hopf algebra $\mathrm{NSym}_{A}$ is both non-commutative and noncocommutative we cannot expect that the antipode is an involution. It turns out that its superposition with the reversal is.

Proposition 40. In the Hopf algebra $\mathrm{NSym}_{A}$ of coloured non-commutative symmetric functions

$$
S \circ r \circ S \circ r=\mathrm{id}
$$

Proof. Notice first that

$$
(S \circ r)\left(E_{I}\right)=S\left(\sum_{J \preccurlyeq I}(-1)^{|I|-\ell(J)} H_{J^{r}}\right)=(-1)^{|I|} \sum_{J \preccurlyeq I}(-1)^{\left|J_{r}\right|-\ell(J)} E_{J}=(-1)^{|I|} H_{I},
$$

where we used equations (3.23), (3.26) and the fact that $\left|J^{r}\right|=|I|$ for $J \preccurlyeq I$. Then in the basis of complete symmetric functions by equations (3.23) and (3.29) we have

$$
(S \circ r \circ S \circ r)\left(H_{I}\right)=(-1)^{|I|}(S \circ r)\left(E_{I}\right)=H_{I},
$$

what concludes the proof. 
We will close this Section by presenting coloured and non-commutative analogs of some classical properties of single-word elementary symmetric functions. The antipode expressions (3.19), in view of equation (3.23), give the following recurrence formulas

$$
E_{\left(a_{i_{1}} a_{i_{2}} \ldots a_{i_{k}}\right)}=\left\{\begin{array}{l}
\left.\sum_{j=0}^{k-1}(-1)^{k-j+1} E_{\left(a_{i_{1}} a_{i_{2}} \ldots a_{i_{j}}\right)} H_{\left(a_{i_{j+1}} \ldots a_{i_{k}}\right)}\right), \\
\sum_{j=1}^{k}(-1)^{j-1} H_{\left(a_{i_{1}} a_{i_{2}} \ldots a_{i_{j}}\right)} E_{\left(a_{i_{j+1}} \ldots a_{i_{k}}\right)},
\end{array}\right.
$$

which start from $E_{1}=1$. Equivalently, the above recurrence relations can be rewritten for $k>0$ as

$$
\sum_{j=0}^{k}(-1)^{j} E_{\left(a_{i_{1}} a_{i_{2}} \ldots a_{i_{j}}\right)} H_{\left(a_{i_{j+1}} \ldots a_{i_{k}}\right)}=\sum_{j=0}^{k}(-1)^{j} H_{\left(a_{i_{1}} a_{i_{2}} \ldots a_{i_{j}}\right)} E_{\left(a_{i_{j+1}} \ldots a_{i_{k}}\right)}=0 .
$$

Finally, we present the coproduct formula for our generalization of the elementary symmetric functions.

Proposition 41. The analog of the coproduct formula (3.18) for coloured non-commutative elementary symmetric functions reads as follows

$$
\Delta\left(E_{I}\right)=\sum_{J \subset I} E_{\widetilde{J}} \otimes E_{\widetilde{I \backslash J}},
$$

which in the case of single-word functions gives

$$
\Delta\left(E_{\left(a_{i_{1}} a_{i_{2}} \ldots a_{i_{k}}\right)}\right)=\sum_{j=0}^{k} E_{\left(a_{i_{j+1}} \ldots a_{i_{k}}\right)} \otimes E_{\left(a_{i_{1}} a_{i_{2}} \ldots a_{i_{j}}\right)} .
$$

Proof. It is enough to prove the second equation, because then Proposition 37 implies the first one. Since it holds for $k=1$, then we can start induction by applying the coproduct operation on the recurrence (3.31). Using the homomorphism property of the comultiplication we can expand corresponding expressions and collect coefficients at various terms of consecutive degrees on the right hand side of the tensor product sign. By the recurrence relations (3.31) most of them vanishes, and what remains gives equation (3.34).

Example 42. To calculate the coproduct of $E_{(a b)}=E_{(a)} H_{(b)}-H_{(a b)}$ first notice that

$\Delta\left(E_{(a b)}\right)=\left(E_{(a)} \otimes 1+1 \otimes E_{(a)}\right)\left(H_{(b)} \otimes 1+1 \otimes H_{(b)}\right)-\left(H_{(a b)} \otimes 1+H_{(a)} \otimes H_{(b)}+1 \otimes H_{(a b)}\right)$.

There is only one term $(\ldots) \otimes 1$ of the right degree zero. Its coefficient is $E_{(a)} H_{(b)}-H_{(a b)}=$ $E_{(a b)}$. There are two terms $(\ldots) \otimes E_{(a)}$ and $(\ldots) \otimes H_{(b)}$ of the right degree one. The coefficient of the first one equals $H_{(b)}=E_{(b)}$, while the coefficient of the second term reads $E_{(a)}-H_{(a)}=0$. Finally, the coefficients of the right degree two have on the left $1 \otimes(\ldots)$ and sum up to $E_{(a)} H_{(b)}-H_{(a b)}=E_{(a b)}$.

Remark 43. Notice that, contrary to the unary (monochromatic) case $|A|=1$, for $|A|>1$ the coproduct formulas for single-word coloured non-commutative complete and elementary symmetric functions are not the same. 


\section{Coloured quasi-symmetric functions}

In this Section we study basic properties of the graded dual to the Hopf algebra $\mathrm{NSym}_{A}$, which we later will call the Hopf algebra of coloured quasi-symmetric functions, and denote by $\mathrm{QSym}_{A}$. In particular, we introduce the dual basis to complete function basis $\left(H_{I}\right)$, which will be called later the basis of coloured monomial quasi-symmetric functions. Then we will provide a realization of the algebra $\mathrm{QSym}_{A}$ in terms of series of bounded degree with partially commuting variables.

\subsection{The graded dual of $\operatorname{NSym}_{A}$}

In the the graded dual $\left(\mathrm{NSym}_{A}\right)^{g r}$ of the Hopf algebra of $A$-coloured non-commutative symmetric functions, by $\left(H_{I}^{*}\right)$ denote the dual basis to the basis $\left(H_{J}\right)$ of complete symmetric functions

$$
\left\langle H_{I}^{*}, H_{J}\right\rangle=\delta_{I, J}
$$

The dual to the concatenation product (3.16) is the deconcatenation coproduct $\delta$, which is given by

$$
\begin{aligned}
\delta\left(H_{I}^{*}\right) & =\sum_{I=J \cdot K} H_{J}^{*} \otimes H_{K}^{*} \quad \text { i.e. } \\
\delta\left(H_{\left(w_{1}, w_{2}, \ldots w_{k}\right)}^{*}\right) & =\sum_{j=0}^{k} H_{\left(w_{1}, w_{2}, \ldots w_{j}\right)}^{*} \otimes H_{\left(w_{j+1}, \ldots w_{k}\right)}^{*},
\end{aligned}
$$

In particular, elements of the dual basis indexed by single-word sentences are primitive elements of the coproduct.

The product in $\left(\mathrm{NSym}_{A}\right)^{g r}$ can be defined directly by dualization of the pruning coproduct of tall trees described in the basis of complete functions by formula (3.16). Equivalently, it can be described in terms of the original grafting product of trees and the dual to the injection map $\mathrm{NSym}_{A} \hookrightarrow \mathbb{k} T_{A}$. It is therefore restriction of the grafting product from ROC-trees to tall trees, i.e. we can graft planted tall trees at the root or on the top of another such tree, with the restriction that two trees cannot be grafted on the same top.

The geometric procedure on the level of trees is the same as that in the monochromatic case, so we keep the same name and symbol of the quasi-shuffle product. Quasi-shuffle $I \stackrel{Q}{\amalg} J$ of two sentences $I=\left(u_{1}, u_{2}, \ldots, u_{k}\right)$ and $J=\left(v_{1}, v_{2}, \ldots, v_{m}\right)$ is thus the sum of shuffles of components $u_{i}$ and $v_{j}$ of $I$ and $J$, where in addition we may replace any number of pairs of consecutive words $u_{i}, v_{j}$ in the shuffle by their concatenation $u_{i} v_{j}$. It can be represented, compare with [51], by a path in the lattice of size $k \times m$ from the left bottom corner to the right top corner with horizontal steps $(1,0)$ representing words $u_{i}$, vertical steps $(0,1)$ representing words $v_{j}$ and oblique steps $(1,1)$ representing words $u_{i} v_{j}$; see Figure 11 for an example.

Therefore we have (compare with equation (2.30))

$$
H_{I}^{*} H_{J}^{*}=\sum_{K} H_{K}^{*}
$$




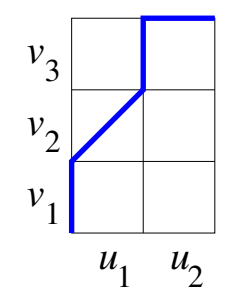

Figure 11: The lattice for quasi-shuffle product of $\left(u_{1}, u_{2}\right)$ and $\left(v_{1}, v_{2}, v_{3}\right)$ with path representing the summand $\left(v_{1}, u_{1} v_{2}, v_{3}, u_{2}\right)$

where $K$ can be obtained as a summand in quasi-shuffle of $I$ and $J$. By dualizing the coproduct formula (3.16) we can see that there exists weak sentence $J^{\prime} \subset K$ such that $J=\widetilde{J^{\prime}}$ and $I=\widetilde{K \backslash J^{\prime}}$.

Remark 44. Similarly to the quasi-shuffle product of compositions, the quasi-shuffle of sentences can be defined recursively for all sentences $I, J$ and all non-empty words $u, v$ by

$$
\begin{gathered}
I \stackrel{Q}{\uplus} 1=1 \stackrel{Q}{\longleftarrow} I=I, \\
((u) \cdot I) \stackrel{Q}{\amalg}((v) \cdot J)=(u) \cdot(I \stackrel{Q}{\sqcup}((v) \cdot J))+(v) \cdot(((u) \cdot I) \stackrel{Q}{\sqcup} J)+(u v) \cdot(I \stackrel{Q}{\amalg} J) .
\end{gathered}
$$

Example 45. The quasi-shuffle product of two tall trees takes the form given in Figure 12, and the product of corresponding monomial functions reads

$$
H_{(a, b)}^{*} H_{(b)}^{*}=2 H_{(a, b, b)}^{*}+H_{(a, b b)}^{*}+H_{(a b, b)}^{*}+H_{(b, a, b)}^{*},
$$

compare also with Figure 7 describing the asymmetric shuffle product of the same trees.

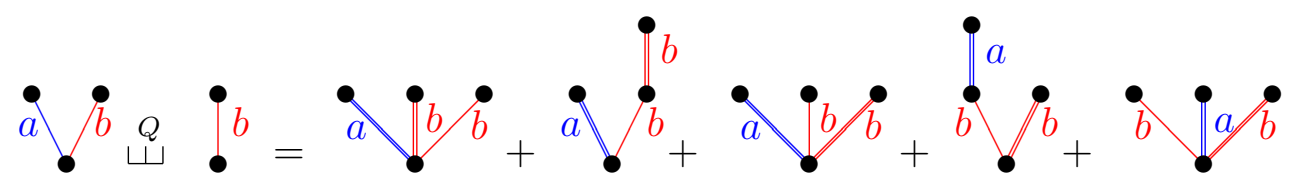

Figure 12: The quasi-shuffle product of two tall trees as given in equation (4.4); grafted planted tall trees are thickened

Remark 46. We have equipped the space of sentences over $A$ with the dual Hopf algebra structure, graded dual to that described in Section 3.1, with quasi-shuffle product and deconcatenation coproduct. Being dual to non-commutative and non-cocommutative Hopf algebra the new algebra, for $|A|>1$, is also both non-commutative and noncocommutative.

Let us calculate the number of quasi-shuffle paths with prescribed number of oblique steps, which will be used in Section 6.3. 

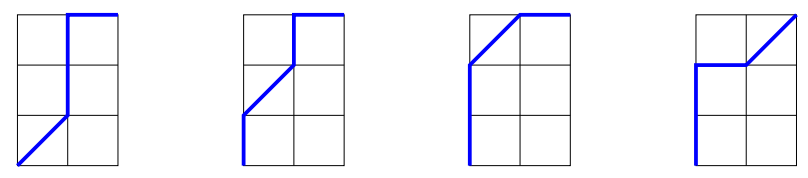

Figure 13: Four quasi-shuffle paths in $2 \times 3$ lattice which have the same shuffle part. The difference is in location of the $(1,1)$ segment which can be placed at any vertex of the contracted path

Proposition 47. The number of quasi-shuffle paths in $k \times m$ lattice with exactly $i$ oblique steps $(1,1), i=0,1, \ldots, \min \{k, m\}$, equals

$$
\left(\begin{array}{c}
k+m-2 i \\
k-i
\end{array}\right)\left(\begin{array}{c}
k+m-i \\
i
\end{array}\right)=\left(\begin{array}{c}
k \\
i
\end{array}\right)\left(\begin{array}{c}
k+m-i \\
k
\end{array}\right)=\left(\begin{array}{c}
m \\
i
\end{array}\right)\left(\begin{array}{c}
k+m-i \\
m
\end{array}\right) .
$$

Proof. Two quasi-shuffle paths are called to have the same shuffle part if they coincide after contracting all the oblique segments $(1,1)$; see Figure 13 for an example. Decomposition of the set of paths with exactly $i$ oblique segments into disjoint classes having the same shuffle part, and then fixing location of the segments, gives the first part of formula (4.5). Two other expressions, which can be derived by simple algebra, also have combinatorial interpretation. The second/third one means that the path can be encoded by first fixing columns/rows for the oblique segments $(1,1)$, and then by choosing which steps of the path are vertical/horizontal segments $(0,1) /(1,0)$.

\subsection{Generalized quasi-symmetric functions}

For each colour $a \in A$, let $x_{a}=\left(x_{a, 1}, x_{a, 2}, x_{a, 3}, \ldots\right)$ denote infinite totally ordered set of variables, each of degree 1 , define also $x_{A}=\bigcup_{a \in A} x_{a}$. We assume partial commutativity of the variables, i.e. within each set the variables commute, but for different colours commutativity is allowed for different second indices only

$$
x_{a, i} x_{b, j}=x_{b, j} x_{a, i} \quad \text { for } \quad i \neq j, \quad a, b \in A,
$$

We will consider a subset $\operatorname{QSym}_{A}$ of the algebra $\mathbb{k}\left[x_{A}\right]$ of series of bounded degree with natural multiplication, which can be described as follows. Due to partial commutativity any monomial in variables $x_{a, i}$ can be uniquely reordered in such a way that the second indices of variables form weakly increasing (finite) sequence, say $i_{1} \leqslant i_{2} \leqslant \ldots \leqslant i_{k}$. Given word $w=a_{i_{1}} a_{i_{2}} \ldots a_{i_{k}}$ and given $j \in \mathbb{N}$, by $x_{w, j}$ denote monomial of degree $|w|$

$$
x_{w, j}=x_{a_{i_{1}}, j} x_{a_{i_{2}}, j} \ldots x_{a_{i_{k}, j}}
$$

for example $x_{a b b, 2}=x_{a, 2} x_{b, 2}^{2}$. From the other side, given such a reordered monomial $x_{w_{1}, j_{1}} x_{w_{2}, j_{2}} \ldots x_{w_{m}, j_{m}}$ with $j_{1}<j_{2}<\cdots<j_{m}$ by its sentence we mean $\left(w_{1}, w_{2}, \ldots, w_{m}\right)$. A formal series belongs to $\mathrm{QSym}_{A}$ when its coefficients in front of monomials with the same sentence coincide. 
It is easy to see that the set $\mathrm{QSym}_{A}$ is in fact linear space with basis indexed by sentences. In fact, given sentence $I=\left(w_{1}, w_{2}, \ldots, w_{m}\right)$, by $M_{I}$ denote the infinite series of the finite degree $|I|$

$$
M_{I}=\sum_{1 \leqslant j_{1}<j_{2}<\cdots<j_{m}} x_{w_{1}, j_{1}} x_{w_{2}, j_{2}} \ldots x_{w_{m}, j_{m}}
$$

which will be called a coloured monomial quasi-symmetric function.

Example 48. Consider product of two such series

$$
M_{(a, b)}=x_{a, 1} x_{b, 2}+x_{a, 1} x_{b, 3}+x_{a, 2} x_{b, 3}+\cdots \quad \text { and } \quad M_{(b)}=x_{b, 1}+x_{b, 2}+x_{b, 3}+\cdots,
$$

which after the reordering reads

$$
\begin{aligned}
& M_{(a, b)} M_{(b)}=\left(x_{a, 1} x_{b, 2}+x_{a, 1} x_{b, 3}+x_{a, 2} x_{b, 3}+\cdots\right)\left(x_{b, 1}+x_{b, 2}+x_{2,3}+\cdots\right)= \\
= & \left(x_{a, 1} x_{b, 1} x_{b, 2}+\cdots\right)+\left(x_{a, 1} x_{b, 2} x_{b, 2}+\cdots\right)+2\left(x_{a, 1} x_{b, 2} x_{b, 3}+\cdots\right)+\left(x_{b, 1} x_{a, 2} x_{b, 3}+\cdots\right)= \\
= & M_{(a b, b)}+M_{(a, b b)}+2 M_{(a, b, b)}+M_{(b, a, b)},
\end{aligned}
$$

and compare with Example 45 or Figure 12.

Proposition 49. The subspace $\mathrm{QSym}_{A}$ of $\mathbb{k}\left[x_{A}\right]$ spanned by the series $\left(M_{I}\right)$ is a subalgebra isomorphic to $\left(\mathrm{NSym}_{A}\right)^{\text {gr }}$ with the isomorphism given by $M_{I} \leftrightarrow H_{I}^{*}$.

Proof. Multiplication of two monomials with different second indices gives monomials with shuffled words. When second indices of two words of both monomials coincide then in the multiplication and reordering procedure the words will be concatenated.

To define the coproduct $\delta$ in $\mathrm{QSym}_{A}$ we use the doubling variables trick described in Section 2.3, i.e. to the set of variables $x_{A}$ we add its copy $y_{A}$. We place the new variables after the old ones, what in particular implies that the new and old variables commute and allows to separate the variables in the reordering process.

Example 50. Applying the doubling variable procedure to $M_{(a b, b)}=x_{a, 1} x_{b, 1} x_{b, 2}+\cdots$ we obtain

$$
\begin{aligned}
x_{a, 1} x_{b, 1} x_{b, 2}+\ldots & \mapsto x_{a, 1} x_{b, 1} x_{b, 2}+\cdots+x_{a, 1} x_{b, 1} y_{b, 1}+\cdots+y_{a, 1} y_{b, 1} y_{b, 2}+\cdots= \\
& =M_{(a b, b)}(x)+M_{(a b)}(x) M_{(b)}(y)+M_{(a b, b)}(y),
\end{aligned}
$$

getting this way

$$
\delta\left(M_{(a b, b)}\right)=M_{(a b, b)} \otimes 1+M_{(a b)} \otimes M_{(b)}+1 \otimes M_{(a b, b)} .
$$

Proposition 51. The algebra isomorphism described in Proposition 49 is the Hopf algebra isomorphism. In particular the deconcatenation coproduct in $\left(\mathrm{NSym}_{A}\right)^{g r}$ can be realized by the variables doubling method in $\mathrm{QSym}_{A}$

$$
\begin{aligned}
\delta\left(M_{I}\right) & =\sum_{I=J . K} M_{J} \otimes M_{K} \quad \text { i.e. } \\
\delta\left(M_{\left(w_{1}, w_{2}, \ldots w_{k}\right)}\right) & =\sum_{j=0}^{k} M_{\left(w_{1}, w_{2}, \ldots w_{j}\right)} \otimes M_{\left(w_{j+1}, \ldots w_{k}\right)} .
\end{aligned}
$$


Proof. It is enough to consider how the variables doubling procedure works for the monomial $x_{w_{1}, 1} x_{w_{2}, 2} \ldots x_{w_{m}, m}$. Finiteness of the size of the sentence assures finite sum decomposition.

Remark 52. There is no need to check coassociativity of the coproduct in $\mathrm{QSym}_{A}$ or its compatibility with the (quasi-shuffle) product, because this holds by definition of $\left(\mathrm{NSym}_{A}\right)^{g r}$.

Finally let us present the formula for the antipode $S^{*}$ in the monomial basis of $\mathrm{QSym}_{A}$. By dualizing the corresponding formula (3.17) for the antipode $S$ in the complete basis of $\mathrm{NSym}_{A}$ we get

$$
S^{*}\left(M_{I}\right)=(-1)^{\ell(I)} \sum_{J^{r} \succcurlyeq I} M_{J}
$$

which for unary words (i.e. usual compositions) reduce to that found in [56, 27].

Example 53. By the equation (4.10)

$$
S^{*}\left(M_{(a b, c)}\right)=M_{(c, a b)}+M_{(a b c)} .
$$

By the coproduct formula

$$
\delta\left(M_{(a b, c)}\right)=1 \otimes M_{(a b, c)}+M_{(a b)} \otimes M_{(c)}+M_{(a b, c)} \otimes 1
$$

and the recurrence (2.6) it should be equal to

$$
-M_{(a b, c)}-S^{*}\left(M_{(a b)}\right) M_{(c)}=-M_{(a b, c)}+M_{(a b)} M_{(c)}=-M_{(a b, c)}+M_{(a b, c)}+M_{(c, a b)}+M_{(a b c)} .
$$

Remark 54. Notice that, contrary to the unary case [34], we cannot sum up in (4.10) with respect to $J \succcurlyeq I^{r}$. Such a summation in the above Example would give incorrect result $M_{(c, a b)}+M_{(c a b)}$.

By dualizing equations (3.20) define the operations of reversal and complement in the monomial basis $\left(M_{I}\right)$

$$
r\left(M_{I}\right)=M_{I^{r}}, \quad c\left(M_{I}\right)=M_{I^{c}},
$$

and extend them to QSym $_{A}$ by linearity.

\section{The fundamental basis of $\operatorname{QSym}_{A}$ and its dual basis in $\operatorname{NSym}_{A}$}

In this Section we define and study another basis of the Hopf algebra of coloured quasisymmetric functions $\mathrm{QSym}_{A}$, which is the analog of the fundamental basis $[71,27]$. Then we consider the dual basis in $\mathrm{NSym}_{A}$ to the coloured fundamental functions, which can be called the basis of coloured ribbon non-commutative Schur functions. 


\subsection{Fundamental coloured quasi-symmetric functions}

Define the fundamental coloured quasi-symmetric functions indexed by sentences as

$$
F_{I}=\sum_{J \preccurlyeq I} M_{J}
$$

By properties of the Moebius function (3.1) of the poset of sentences one can invert the above relation

$$
M_{I}=\sum_{J \preccurlyeq I}(-1)^{\ell(J)-\ell(I)} F_{J},
$$

what shows that the fundamental functions form a linear basis in QSym $_{A}$.

Let us find expressions for the coproduct, product and the antipode of $\mathrm{QSym}_{A}$ in the fundamental basis.

Proposition 55. The coproduct in the fundamental basis reads

$$
\delta\left(F_{I}\right)=\sum_{\substack{K^{\prime} \cdot K^{\prime \prime}=I \\ K^{\prime} \odot K^{\prime \prime}=I}} F_{K^{\prime}} \otimes F_{K^{\prime \prime}}
$$

where the summation is over pairs of sentences which give the indexing sentence I by concatenation or the near-concatenation.

Proof. By definition (5.1) and coproduct formula (4.9) in the monomial basis, and grouping terms we obtain

$$
\delta\left(F_{I}\right)=\sum_{J \preccurlyeq I}\left(\sum_{J^{\prime} . J^{\prime \prime}=J} M_{J^{\prime}} \otimes M_{J^{\prime \prime}}\right)=\sum_{\left(K^{\prime}, K^{\prime \prime}\right)} F_{K^{\prime}} \otimes F_{K^{\prime \prime}},
$$

where the sum is over the pairs $\left(K^{\prime}, K^{\prime \prime}\right)$ which give splitting of $I$ into two parts. The segmentation may be either between words of $I$ or in the middle of a word. The first case gives $I=K^{\prime} \cdot K^{\prime \prime}$, while the second one gives $I=K^{\prime} \odot K^{\prime \prime}$.

Example 56. The deconcatenation coproduct of the fundamental function $F_{(a b, c)}=$ $M_{(a b, c)}+M_{(a, b, c)}$ is given by

$$
\delta\left(F_{(a b, c)}\right)=1 \otimes F_{(a b, c)}+F_{(a)} \otimes F_{(b, c)}+F_{(a b)} \otimes F_{(c)}+F_{(a b, c)} \otimes 1 .
$$

In order to describe multiplication in the fundamental basis notice that in multiplying $F_{I}$ and $F_{J}$ we multiply $M_{I^{\prime}}$ and $M_{J}$ for any $I^{\prime} \preccurlyeq I$ and $J^{\prime} \preccurlyeq J$. Then we group monomial functions into the fundamental ones. This procedure leads to definition of the fundamental shuffle $I \stackrel{F}{\uplus} J$ of sentences described as follows:

1. perform ordinary shuffle $\uplus$ of letters of maximal words of both sentences,

2. concatenate neighboring letters of words of $I$, and concatenate neighboring letters of words of $J$, 
3. concatenate pairs of neighboring subwords of words of $I$ and $J$ (in this order), which gives directly the desired formula.

Proposition 57. Multiplication of two fundamental functions is given by

$$
F_{I} \cdot F_{J}=\sum F_{K}
$$

where sentence $K$ is a summand of $I \stackrel{F}{\amalg} J$.

Example 58. Let us perform the procedure in order to calculate $(a b) \stackrel{F}{\amalg}(c, d)$. To remember, at each step of the procedure, the origin of letters of the second word we mark them by the tilde sign. At subsequent steps we obtain:

1. $(a, b, \tilde{c}, \tilde{d})+(a, \tilde{c}, b, \tilde{d})+(a, \tilde{c}, \tilde{d}, b)+(\tilde{c}, a, b, \tilde{d})+(\tilde{c}, a, \tilde{d}, b)+(\tilde{c}, \tilde{d}, a, b)$

2. $(a b, \tilde{c}, \tilde{d})+(a, \tilde{c}, b, \tilde{d})+(a, \tilde{c}, \tilde{d}, b)+(\tilde{c}, a b, \tilde{d})+(\tilde{c}, a, \tilde{d}, b)+(\tilde{c}, d, a b)$

3. $(a b \tilde{c}, \tilde{d})+(a \tilde{c}, b \tilde{d})+(a \tilde{c}, \tilde{d}, b)+(\tilde{c}, a b \tilde{d})+(\tilde{c}, a \tilde{d}, b)+(\tilde{c}, \tilde{d}, a b)$,

what gives

$$
F_{(a b)} \cdot F_{(c, d)}=F_{(a b c, d)}+F_{(a c, b d)}+F_{(a c, d, b)}+F_{(c, a b d)}+F_{(c, a d, b)}+F_{(c, d, a b)} .
$$

Remark 59. In passing to the unary alphabet we obtain the corresponding multiplication formula for the fundamental quasi-symmetric functions [72, 34]. Because the same structure of posets of compositions and of sentences the proof presented there can be transferred also to our context. One has to label letters of the maximal words of the two sentences by integers, whose descent sets model the separation of letters into words.

Proposition 60. The antipode in the fundamental basis is given by

$$
S^{*}\left(F_{I}\right)=(-1)^{|I|} r\left(F_{I^{c}}\right) .
$$

Proof. Expanding the fundamental function in the monomial basis and using of (4.10) we obtain

$$
S^{*}\left(F_{I}\right)=\sum_{J \preccurlyeq I} S^{*}\left(M_{J}\right)=\sum_{J \preccurlyeq I}(-1)^{\ell(J)} \sum_{K \succcurlyeq J} M_{K^{r}}=\sum_{K} M_{K^{r}} \sum_{\substack{J \preccurlyeq K \\ J \preccurlyeq I}}(-1)^{\ell(J)},
$$

where the last sum is over sentences $J$ which refine simultaneously $K$ and $I$. By properties of the Moebius function of the poset of such refinements the sum doesn't vanish only if the poset consists of one element only, which must be therefore the sentence of one-letter words. In this case $\ell(J)=|I|$ and $K$ must be a refinement of the complement of $I$, i.e. $K \preccurlyeq I^{c}$. This gives the corresponding version of monochromatic formulas of $[27,56]$

$$
S^{*}\left(F_{I}\right)=\sum_{K \preccurlyeq I^{c}}(-1)^{|I|} r\left(M_{K}\right)=(-1)^{|I|} r\left(F_{I^{c}}\right) .
$$


Example 61. In order to directly calculate the antipode of $F_{(a b, c)}=M_{(a b, c)}+M_{(a, b, c)}$ we first find from equation (4.10)

$$
S^{*}\left(M_{(a b, c)}\right)=M_{(c, a b)}+M_{(a b c)}, \quad S^{*}\left(M_{(a, b, c)}\right)=-\left(M_{(c, b, a)}+M_{(c, a b)}+M_{(b c, a)}+M_{(a b c)}\right),
$$

which summed up give $S^{*}\left(F_{(a b, c)}\right)=-\left(M_{(c, b, a)}+M_{(b c, a)}\right)=-r\left(F_{(a, b c)}\right)$ in agreement with (5.8).

Remark 62. Notice that in general $r\left(F_{I}\right) \neq F_{I^{r}}$, for example

$$
r\left(F_{(a, b c)}\right)=M_{(b c, a)}+M_{(c, b, a)}, \quad \text { while } \quad F_{(a, b c)^{r}}=M_{(b c, a)}+M_{(b, c, a)} .
$$

Corollary 63. By involutivity of reversal and complement operations we directly obtain the dual counterpart of formula (3.28)

$$
r \circ S^{*} \circ r \circ S^{*}=\text { id. }
$$

As an exercise we recommend for the interested Reader to perform the calculation in the monomial basis.

\subsection{Coloured ribbon non-commutative Schur functions}

Consider the basis $\left(R_{I}\right)$ in $\mathrm{NSym}_{A}$ dual to the fundamental basis $\left(F_{I}\right)$ in $\mathrm{QSym}_{A}$

$$
\left\langle F_{I}, R_{J}\right\rangle=\delta_{I, J}
$$

In the monochromatic case such basis was introduced in [32] as a non-commutative analog of the ribbon Schur functions [53].

Proposition 64 . The relation between the complete basis $\left(H_{I}\right)$ and the ribbon basis $\left(R_{I}\right)$ is given by

$$
\begin{aligned}
H_{I} & =\sum_{J \succcurlyeq I} R_{J}, \\
R_{I} & =\sum_{J \succcurlyeq I}(-1)^{\ell(J)-\ell(I)} H_{J} .
\end{aligned}
$$

Proof. For the first assertion, note that

$$
H_{I}=\sum_{J}\left\langle L_{J}, H_{I}\right\rangle R_{J}=\sum_{J} \sum_{K \preccurlyeq J}\left\langle M_{K}, H_{I}\right\rangle R_{J}=\sum_{J \succcurlyeq I} R_{J} .
$$

The second assertion follows from the first one by inclusion-exclusion.

Corollary 65. The dual version of Proposition 55 gives the multiplication formula in the ribbon basis

$$
R_{I} R_{J}=R_{I \cdot J}+R_{I \odot J}
$$


Corollary 66. The dual version of Proposition 57 gives the comultiplication in the ribbon basis

$$
\Delta\left(R_{I}\right)=\sum_{J, K}\left\langle F_{J \sqcup K}^{F}, R_{I}\right\rangle R_{J} \otimes R_{K}
$$

Example 67. Both calculations using the complete basis expansion (5.15) with the corresponding coproduct formula (3.16) or the above Corollary and definition of the fundamental shuffle give

$\Delta\left(R_{(a b, c)}\right)=1 \otimes R_{(a b, c)}+R_{(a)} \otimes R_{(b, c)}+R_{(c)} \otimes R_{(a b)}+R_{(a, c)} \otimes R_{(b)}+R_{(a c)} \otimes R_{(b)}+R_{(a b, c)} \otimes 1$.

In particular, let us present the fundamental shuffle products of the non-trivial sentences which give the sentence $(a b, c)$ as a summand (typed in boldface):

$$
\begin{aligned}
(a) \stackrel{F}{\uplus}(b, c) & =(\boldsymbol{a} \boldsymbol{b}, \boldsymbol{c})+(b, a c)+(b, c, a), \\
(c) \stackrel{F}{\amalg}(a b) & =(c a b)+(a, c b)+(\boldsymbol{a} \boldsymbol{b}, \boldsymbol{c}), \\
(a, c) \stackrel{F}{\uplus}(b) & =(a, c b)+(\boldsymbol{a b}, \boldsymbol{c})+(b, a, c), \\
(a c) \stackrel{F}{\amalg}(b) & =(a c b)+(\boldsymbol{a} \boldsymbol{b}, \boldsymbol{c})+(b, a c) .
\end{aligned}
$$

In the monochromatic case there exists [34] a convenient formula, of the form (5.8), expressing the coproduct in the ribbon basis. Because in the coloured case taking refinements does not commute with reversal we can provide only the following result.

Corollary 68. The dual version of equation (5.8) reads as follows

$$
S \circ r\left(R_{I}\right)=(-1)^{|I|} R_{I^{c}} .
$$

Proof. This can be shown by dualization of equation (5.8). Let us provide also direct proof. By expressing the ribbon basis in the complete one, and by using the corresponding formula for the antipode we obtain

$$
\begin{aligned}
S \circ r\left(R_{I}\right) & =\sum_{J \succcurlyeq I}(-1)^{\ell(J)-\ell(I)} S\left(H_{J^{r}}\right)=\sum_{J \succcurlyeq I}(-1)^{\ell(J)-\ell(I)} \sum_{K \preccurlyeq J^{r r}}(-1)^{\ell(K)} H_{K}= \\
& =\sum_{K}(-1)^{\ell(K)} H_{K} \sum_{\substack{J \succcurlyeq K \\
J \succcurlyeq I}}(-1)^{\ell(J)-\ell(I)}=(-1)^{|I|} \sum_{K \succcurlyeq I^{c}}(-1)^{\ell(K)-\ell\left(I^{c}\right)} H_{K}=(-1)^{|I|} R_{I^{c}} .
\end{aligned}
$$

Above we sum up with respect to all sentences $K$ having the same maximal word $w(I)$, and the inner sum is over sentences $J$ which coarsen simultaneously $K$ and $I$. This sum doesn't vanish only when this poset of sentences is trivial what happens only if $K$ coarsens the complement of $I$. In this case $J$ is the single-word sentence, thus $\ell(J)=1$. To conclude the calculation we notice that $\ell(I)+\ell\left(I^{c}\right)=|I|-1$. 


\section{Formal series of trees and coloured non-commutative power sum functions}

Up to now we considered the duality problem for infinite-dimensional Hopf algebras in the graded case only. Another option to tackle the problem is to define [1, 74] the restricted (or Sweedler's) dual of $\mathcal{H}$ which is the subspace $\mathcal{H}^{\circ} \subset \mathcal{H}^{*}$ consisting of all linear maps $f$ that satisfy one of equivalent conditions:

1. $\Delta_{\mathcal{H}^{*}}(f) \in \mathcal{H}^{*} \otimes \mathcal{H}^{*}$,

2. $\operatorname{ker}(f)$ contains an ideal (left, right or two-sided) of $\mathcal{H}$ that has finite codimension.

Define a left action $\rightarrow$ of $\mathcal{H}$ on $\mathcal{H}^{*}$ as the transpose of right multiplication on $\mathcal{H}$

$$
\langle a \rightarrow f, b\rangle=\langle f, b a\rangle \quad f \in \mathcal{H}^{*}, \quad a, b \in \mathcal{H} .
$$

Then $\mathcal{H} \rightarrow f$ is a subspace of $\mathcal{H}^{*}$, and the condition $f \in \mathcal{H}^{\circ}$ is equivalent to

(3) $\operatorname{dim}(\mathcal{H} \rightarrow f)<\infty$.

Remark 69. One can define also a right action $<$ of $\mathcal{H}$ on $\mathcal{H}^{*}$ as the transpose of left multiplication on $\mathcal{H}$. Then condition (3) can be equivalently stated as finite-dimensionality of $f<\mathcal{H}$ or finite-dimensionality of $\mathcal{H} \rightarrow f<\mathcal{H}$.

\subsection{Formal series of ROC trees}

In this Section we consider power series of trees as the linear dual to space of rooted ordered coloured (by $A$ ) trees $\mathbb{k} T_{A}$. A formal tree series $F$ is function $T_{A} \rightarrow \mathbb{k}$ extended to $\mathbb{k} T_{A}$ by linearity. The image by $F \in\left(\mathbb{k} T_{A}\right)^{*}$ of a tree $t \in T_{A}$ is denoted by $\langle F, t\rangle$ and is called the coefficient of $t$ in $T$. The support of $F$ is the subset of $T_{A}$

$$
\operatorname{supp}(F)=\left\{t \in T_{A} \mid\langle F, t\rangle \neq 0\right\} .
$$

Polynomials $\mathbb{k} T_{A} \subset\left(\mathbb{k} T_{A}\right)^{*}$ are embedded naturally as series with finite support. Usually one writes

$$
F=\sum_{t \in T_{A}}\langle F, t\rangle t
$$

remembering that the sum

$$
\mathbb{k} T_{A} \ni P \mapsto\langle F, P\rangle=\sum_{t \in T_{A}}\langle F, t\rangle\langle P, t\rangle,
$$

has a finite support.

Remark 70. Notice that, by the standard coding of trees by (coloured) Dyck words [72], any such series of trees can be interpreted as a series of words within theory of noncommutative power series [68]. As it was mentioned in Corollary 22, our description of the algebra $\mathrm{NSym}_{A}$ can be stated in terms of a certain context-free language. 
Remark 71 . If $\mathbb{k}$ is equipped with discrete topology, then the set of formal tree series can be equipped with the product topology. A sequence of its elements converges only if for each tree the corresponding coefficient stabilizes.

Actually, two products of such series are well defined:

- the extension of the concatenation product "." of trees (i.e. the Cauchy product of series)

$$
F . G=\sum_{t \in T_{A}}\left(\sum_{t^{\prime} \cdot t^{\prime \prime}}\left\langle F, t^{\prime}\right\rangle\left\langle G, t^{\prime \prime}\right\rangle\right) t
$$

- the extension of the grafting product $\stackrel{T}{\amalg}$ of trees

$$
F \stackrel{T}{\uplus} G=\sum_{t^{\prime}, t^{\prime \prime} \in T_{A}}\left\langle F, t^{\prime}\right\rangle\left\langle G, t^{\prime \prime}\right\rangle t^{\prime} \stackrel{T}{\uplus} t^{\prime \prime} .
$$

The tensor product $F \otimes G \in\left(\mathbb{k} T_{A}\right)^{*} \otimes\left(\mathbb{k} T_{A}\right)^{*}$ of two series reads

$$
F \otimes G=\sum_{t, s \in T_{A}}\langle F, t\rangle\langle G, s\rangle t \otimes s .
$$

The deconcatenation coproduct extended from tree polynomials to series

$$
\delta(F)=\sum_{t, s \in T_{A}}\langle F, t . s\rangle t \otimes s,
$$

is in general an element of $\left(\mathbb{k} T_{A} \otimes \mathbb{k} T_{A}\right)^{*}$. A series $F$ which allows for finite decomposition

$$
\delta(F)=\sum_{i=1}^{r} G_{i} \otimes H_{i} \in\left(\mathbb{k} T_{A}\right)^{*} \otimes\left(\mathbb{k} T_{A}\right)^{*}, \quad \text { with } \quad G_{i}, H_{i} \in\left(\mathbb{k} T_{A}\right)^{*},
$$

is an element of the restricted dual $\left(\mathbb{k} T_{A}, ., \Delta\right)^{\circ}$. The pruning coproduct of a tree series can be defined analogously

$$
\Delta(F)=\sum_{t, s \in T_{A}}\langle F, t \stackrel{T}{\amalg} s\rangle t \otimes s
$$

Let us describe a distinguished example of two of such tree series. By $F^{*}$ let us denote the characteristic series of the set of all ROC trees, i.e. $F^{*}=\sum_{t \in T_{A}} t$, and by $F=\sum_{t \in T_{A}^{\prime}} t$ denote the characteristic series of the subset $T_{A}^{\prime}$ of planted trees. Relations between these series can be written down as follows:

(i) an arbitrary planted tree is obtained by action of the operator $B_{i}^{+}, i=1,2, \ldots,|A|$, on the corresponding tree

$$
F=\sum_{i=1}^{|A|} B_{i}^{+}\left(F^{*}\right)
$$


(ii) any non-trivial tree can be uniquely decomposed into concatenation product of planted trees (this justifies our notation)

$$
F^{*}=\bullet+F+F \cdot F+F \cdot F \cdot F+\cdots=\bullet+F \cdot F^{*} .
$$

Remark 72. Equations (6.10)-(6.11) expresses the standard grammar rules of the $|A|$-th Dyck language [68].

Remark 73. By combining equations (6.10) and (6.11) we obtain a single equation for series $F$

$$
F=\sum_{i=1}^{|A|} B_{i}^{+}\left(\bullet+\sum_{k=1}^{\infty} F^{k}\right)
$$

in the form of the combinatorial Dyson-Schwinger equation $[8,30]$.

Proposition 74. The series $F$ and $F^{*}$ are elements of the restricted dual $\left(\mathbb{k} T_{A}, ., \Delta\right)^{\circ}$ of the Hopf algebra of rooted ordered coloured trees, in particular $F$ is primitive element

$$
\delta(F)=\bullet \otimes F+F \otimes \bullet,
$$

and $F^{*}$ is group-like element, i.e.

$$
\delta\left(F^{*}\right)=F^{*} \otimes F^{*} .
$$

Proof. Equation (6.13) follows by linearity from the analogous result valid for any planted tree $t^{\prime} \in T_{A}^{\prime}$

$$
\delta\left(t^{\prime}\right)=\bullet \otimes t^{\prime}+t^{\prime} \otimes \bullet .
$$

When $t^{\prime} \in T_{A}^{\prime}$ is a planted tree and $t \in T_{A}$ is an arbitrary tree then by Corollary 10

$$
\delta\left(t^{\prime} . t\right)=\bullet \otimes\left(t^{\prime} . t\right)+\left(t^{\prime} \otimes \bullet\right) \cdot \delta(t),
$$

which by linearity leads to the following equation on the level of the corresponding series

$$
\delta\left(F . F^{*}\right)=\bullet \otimes\left(F . F^{*}\right)+(F \otimes \bullet) . \delta\left(F^{*}\right) .
$$

Adding to both sides of the above $\delta(\bullet)=\bullet \otimes \bullet$ and using equation (6.11) we obtain the relation

$$
\delta\left(F^{*}\right)=\bullet \otimes F^{*}+(F \otimes \bullet) . \delta\left(F^{*}\right) .
$$

Its solution with respect to $\delta\left(F^{*}\right)$ is

$$
\delta\left(F^{*}\right)=\left(\bullet \otimes F^{*}\right) \cdot(F \otimes \bullet)^{*}=\left(\bullet \otimes F^{*}\right) \cdot\left(F^{*} \otimes \bullet\right)
$$

and leads directly to (6.14).

Corollary 75. Any series, whose support is a subset of planted trees $T_{A}^{\prime}$ is a primitive element of the restricted dual.

Remark 76. Notice that Corollary 10 implies the following matching condition between the cut comultiplication $\delta$ and the Cauchy product of two series $G$ and $H$ of trees in the restricted dual

$$
\delta(G \cdot H)=\delta(G) \cdot(\bullet \otimes H)+(G \otimes \bullet) \cdot \delta(H)-G \otimes H .
$$




\subsection{Formal series of tall trees}

By $H^{*}=\sum_{I} I$ denote the characteristic series of the set of coloured tall trees (indexed by sentences over $A$ ) and by $H=\sum_{w \in A^{*} \backslash\{1\}}(w)$ denote the characteristic series of planted coloured tall trees (indexed by single word sentences). The planting operator $B_{a}^{+}, a \in A$, acts on single word sentence $(w)$ by forming the single word sentence $(w a)$. Properties of the series can be stated as follows:

(i) an arbitrary planted tall tree is obtained by action of the operator $B_{i}^{+}, i=$ $1,2, \ldots,|A|$ on the trivial tree or on the corresponding smaller planted tall tree

$$
H=\sum_{i=1}^{|A|} B_{i}^{+}(\bullet+H),
$$

(ii) any non-trivial tall tree can be uniquely decomposed into concatenation of planted tall trees

$$
H^{*}=\bullet+H+H . H+H . H . H+\cdots=\bullet+H . H^{*} .
$$

Remark 77. Equation (6.19) is of the combinatorial Dyson-Schwinger form.

The following result can be proven in the same way as the previous Proposition 74.

Proposition 78. With respect to the deconcatenation coproduct $\delta$ the series $H$ is primitive element of the restricted dual

$$
\delta(H)=\bullet \otimes H+H \otimes \bullet
$$

and the series $H^{*}$ is group-like

$$
\delta\left(H^{*}\right)=H^{*} \otimes H^{*}
$$

Remark 79. Actually, for action of the coproduct $\delta$ we should write the series $H$ and $H^{*}$ in terms of the coloured monomial quasi-symmetric functions

$$
\begin{gathered}
H_{Q}=\sum_{w \in A^{*} \backslash\{1\}} M_{(w)}, \quad H_{Q}^{*}=\sum_{I} M_{I}, \\
\delta\left(H_{Q}\right)=1 \otimes H_{Q}+H_{Q} \otimes 1, \quad \delta\left(H_{Q}^{*}\right)=H_{Q}^{*} \otimes H_{Q}^{*} .
\end{gathered}
$$

i.e. as elements of the restricted dual $\left(\mathrm{NSym}_{A}\right)^{\circ}$.

Finally let us present formulas for antipodes of the above series. Because $H_{Q}$ is primitive element then

$$
S^{*}\left(H_{Q}\right)=-H_{Q},
$$

but the antipode of the sum of all coloured monomial quasi-symmetric functions takes also particularly simple form. 
Corollary 80. The action of the antipode $S^{*}$ on the series $H_{Q}^{*}$ reads

$$
S^{*}\left(H_{Q}^{*}\right)=\sum_{w \in A^{*}}(-1)^{|w|} M_{\left(w^{c}\right)}
$$

i.e. is the signed sum of monomial functions indexed by minimal compositions (i.e. by sentences built from single-letter words).

Proof. Decompose the series $H_{Q}^{*}$ into parts indexed by compositions with the same maximal word

$$
H_{Q}^{*}=\sum_{I} M_{I}=\sum_{w \in A^{*}} F_{(w)}
$$

where $F_{(w)}$ is the fundamental function indexed by the corresponding single-word composition, and apply Proposition 60.

Because bases of both algebras $\mathrm{NSym}_{A}$ and $\mathrm{QSym}_{A}$ are indexed by sentences one can apply also the pruning coproduct $\Delta$ on series of tall trees. In particular, we will show that the series $H$ can be considered as an element of the restricted dual $\left(\mathrm{QSym}_{A}\right)^{\circ}$. From now on we use the notation of the theory of the non-commutative coloured symmetric functions.

Proposition 81. The pruning coproduct of the series $H=\sum_{w \in A^{+}} H_{(w)}$, where $A^{+}=$ $A^{*} \backslash\{1\}$ reads

$$
\Delta(H)=1 \otimes H+H \otimes 1+H \otimes H .
$$

Proof. By Corollary 30, for arbitrary one word sentence $(w)$ we have

$$
\Delta\left(H_{(w)}\right)=\sum_{u v=w} H_{(u)} \otimes H_{(v)} .
$$

Therefore, summing up with respect to the arbitrary prefix $(u)$ first, we can write

$$
\Delta\left(\sum_{w \in A^{+}} H_{(w)}\right)=\sum_{w \in A^{+}} \sum_{u v=w} H_{(u)} \otimes H_{(v)}=\sum_{u \in A^{*}} H_{(u)} \otimes\left(\sum_{v \in u^{-1} A^{+}} H_{(v)}\right),
$$

where for any subset $L \subset A^{*}$ by definition [54]

$$
u^{-1} L=\{v \mid u v \in L\}
$$

Because

$$
u^{-1} A^{+}= \begin{cases}A^{+} & u=1 \\ A^{*} & u \in A^{+}\end{cases}
$$

then we obtain

$$
\Delta(H)=1 \otimes H+H \otimes(1+H)
$$

Corollary 82. The series $1+H=\sum_{w \in A^{*}} H_{(w)}$ is group-like element of the restricted dual $\left(\mathrm{QSym}_{A}\right)^{\circ}$. 


\subsection{Non-commutative coloured power sum symmetric functions}

Define the power series

$$
P=\log (1+H)=\sum_{n=1}^{\infty} \frac{(-1)^{n-1}}{n} H^{n},
$$

which exists because $H$ has vanishing constant term [68].

Proposition 83. In the basis of complete homogeneous functions indexed by compositions we have

$$
P=\sum_{I \neq 1} \frac{(-1)^{\ell(I)-1}}{\ell(I)} H_{I}
$$

moreover the series $P$ is primitive with respect to the pruning coproduct

$$
\Delta(P)=1 \otimes P+P \otimes 1 .
$$

Proof. The first part follows directly from the definition of $P$, where we recall that the length $\ell(I)$ of the sentence $I$ is the number of its words. For the second part we provide two proofs. The first one repeats the corresponding reasoning [56] in the monochromatic case. The second proof is of elementary combinatorial nature.

I. By linearity and morphism property of $\Delta$ with respect to the concatenation product, and using Corollary 82 we have

$$
\begin{aligned}
& \Delta(P)=\log [\Delta(1+H)]=\log [(1+H) \otimes(1+H)]=\log [((1+H) \otimes 1) \cdot(1 \otimes(1+H))]= \\
& =\log [(1+H) \otimes 1]+\log [1 \otimes(1+H)] \log [1+H] \otimes 1+1 \otimes \log [1+H]=P \otimes 1+1 \otimes P,
\end{aligned}
$$

where we also used the standard property of logarithm for commuting factors.

II. By the basic coproduct formula (3.16) applied to equation (6.31) we can see that

$$
\Delta(P)=P \otimes 1+1 \otimes P+\sum_{J, K \neq 1} c_{J K} H_{J} \otimes H_{K},
$$

where the coefficient $c_{J K}$ equals

$$
c_{J K}=\sum_{I} \frac{(-1)^{\ell(I)-1}}{\ell(I)},
$$

where we sum with respect to the sentences $I$ which give $J \otimes K$ upon action of the coproduct, i.e. $I$ is a summand in the quasi-shuffle $J \stackrel{Q}{\amalg} K$. Interpretation of such terms as special paths in the lattice $\ell(J) \times \ell(K)$, and application of Proposition 47 implies that the weighted alternating sum we are looking for equals (without losing generality we assume $\ell(K) \geqslant \ell(J))$

$$
\frac{(-1)^{\ell(J)+\ell(K)-1}}{\ell(J)} \sum_{i=0}^{\ell(J)}(-1)^{i}\left(\begin{array}{c}
\ell(J) \\
i
\end{array}\right)\left(\begin{array}{c}
\ell(K)+\ell(J)-i-1 \\
\ell(J)-1
\end{array}\right),
$$

which vanishes by standard application of the inclusion-exclusion principle. 
Finally, we define the coloured non-commutative analogs of the power sum symmetric functions. By splitting series $P$ into parts with the same maximal words, see Corollary 19 , we obtain from equation (6.31)

$$
P=\sum_{w \in A^{+}} P_{(w)}, \quad P_{(w)}=\sum_{I \preccurlyeq(w)} \frac{(-1)^{\ell(I)-1}}{\ell(I)} H_{I} .
$$

Here $|w| P_{(w)}$ are coloured analogs of the non-commutative power sums of the second kind defined in [32]. For trivial sentence define $P_{1}=1$, and for any non-empty sentence $I=\left(w_{1}, w_{2}, \ldots, w_{\ell(I)}\right)$ define

$$
P_{I}=P_{\left(w_{1}\right)} P_{\left(w_{2}\right)} \ldots P_{\left(w_{\ell(I)}\right)}
$$

Proposition 84. Functions $P_{I}$ indexed by sentences form a linear basis of $\mathrm{NSym}_{A}$, in particular

$$
H_{(w)}=\sum_{I \preccurlyeq(w)} \frac{1}{\ell(I) !} P_{I} .
$$

Proof. By the standard relation between exponential and logarithm, valid also for formal non-commuting series, we have

$$
1+\sum_{w \in A^{+}} H_{(w)}=\exp (P)=\sum_{n=0}^{\infty} \frac{1}{n !} P^{n}=\sum_{n=0}^{\infty} \frac{1}{n !}\left(\sum_{w \in A^{+}} P_{(w)}\right)^{n}=1+\sum_{I \neq 1} \frac{1}{\ell(I) !} P_{I},
$$

and formula (6.37) follows from splitting of both sides into sentences with the same maximal word.

Example 85. For $w=a b$ we have

$$
\begin{gathered}
P_{(a b)}=H_{(a b)}-\frac{1}{2} H_{(a)} H_{(b)}, \\
\Delta\left(P_{(a b)}\right)=1 \otimes P_{(a b)}+P_{(a b)} \otimes 1+\frac{1}{2}\left(H_{(a)} \otimes H_{(b)}-H_{(b)} \otimes H_{(a)}\right) .
\end{gathered}
$$

As the above example demonstrates, contrary to the monochromatic/unary case the coloured power sum functions are in general not primitive elements of the Hopf algebra $\mathrm{NSym}_{A}$. However, by splitting equation (6.32) into homogeneous parts we obtain the following weaker result, which provides infinite number of primitive elements of the algebra.

Corollary 86. For $n \in \mathbb{N}$ define $P_{n}=\sum_{|w|=n} P_{(w)}$ then

$$
\Delta\left(P_{n}\right)=1 \otimes P_{n}+P_{n} \otimes 1
$$




\section{Conclusion}

We have defined new generalization $\mathrm{NSym}_{A}$ and $\mathrm{QSym}_{A}$ of the Hopf algebras of noncommutative symmetric and quasi-symmetric functions. In our extensions, bases in both algebras are indexed by sentences over finite alphabet $A$ (the set of colours), and our results reduce to the classical ones for the sunary alphabet $|A|=1$ (the monochromatic reduction). We have presented corresponding analogs of the most pertinent structural elements of the original theory including the description of bases of the complete homogeneous, elementary, monomial, fundamental, the ribbon Schur and the power sum functions. It is interesting that, contrary to the monochromatic/unary case, both algebras are non-commutative and non-cocommutative. We have also found a realization of the algebra $\mathrm{QSym}_{A}$ in terms of power series of bounded degree in partially commuting variables, what justifies its name as coloured quasi-symmetric functions.

In our approach the algebra $\mathrm{NSym}_{A}$ is described as Hopf subalgebra of rooted ordered coloured trees. We study also formal series of such trees within the setting of the restricted duals. This new aspect of the theory deserves deeper studies in relation to the renormalization procedure in quantum field theory, non-commutative integrable systems and context-free languages, and will be developed in another publication. In the literature there are known several generalizations of the non-commutative symmetric and quasi-symmetric functions. We strongly believe that the generalization proposed in our paper, being natural and structurally close to the original theory, will be useful in studying problems in combinatorics and theoretical physics.

\section{Acknowledgements}

I would like to thank the Reviewers for careful reading of the manuscript and for their comments which helped to improve the presentation. The research was supported by National Science Centre, Poland, under grant 2015/19/B/ST2/03575 Discrete integrable systems - theory and applications.

\section{References}

[1] Eiichi Abe, Hopf Algebras, Cambridge University Press, 1980, Cambridge.

[2] Pierre Baumann, Christophe Hohlweg, A Solomon-type epimorphism for MantaciReutenauer's algebra of a wreath product $G \backslash \mathfrak{S}_{n}$, Trans. Amer. Math. Soc. 360 (2008) $1475-1538$.

[3] Andrew Baker, Birgit Richter, Quasisymmetric functions from a topological point of view, Math. Scand. 103 (2008) 208-242.

[4] Chris Berg, Nantel Bergeron, Fanco Saliola, Luis Serrano, Mike Zabrocki, A lift of the Schur and Hall-Littlewwod bases to non-commutative symmetric functions, Cannad. J. Math. 66 (2014) 525-565.

[5] Nantel Bergeron, Christophe Hohlweg, Coloured peak algebras and Hopf algebras, J. Algebraic Combin. 24 (2006) 299-330. 
[6] Jean Berstel, Christophe Reutenauer, Noncommutative rational series with applications, Cambridge University Press, 2010, Cambridge.

[7] Alexander I. Bobenko, Yuri B. Suris, Integrable non-commutative equations on quadgraphs. The consistency approach, Lett. Math. Phys. 61 (2002) 241-254.

[8] David J. Broadhurst, Dirk Kreimer, Exact solutions of Dyson-Schwinger equations for iterated one-loop integrals and propagator-coupling duality, Nucl. Phys. B 600 (2001) 403-422.

[9] Christian Brouder, Alessandra Frabetti, QED Hopf algebras on planar binary trees, J. Algebra 267 (2003) 298-322.

[10] John C. Butcher, An algebraic theory of integration methods, Math. Comput. 26 (1972) 79-106.

[11] Pierre Cartier, Dominique Foata, Problèmes combinatoires de commutation et réarrangements, Lecture Notes in Mathematics 85, Springer-Verlag, Berlin, New York, 1969.

[12] Vyjayanthi Chari, Andrew Pressley, A guide to quantum gropus, Cambridge University Press, Cambridge, 1994.

[13] Alain Connes, Dirk Kreimer, Hopf algebras, renormalization and non-commutative geometry, Commun. Math. Phys. 199 (1998) no. 1, 203-242.

[14] Percy Deift, Integrable systems and combinatorial theory, Notices AMS 47 (2000) 631-640.

[15] Philippe Di Francesco, Rinat Kedem, Non-commutative integrability, paths and quasideterminants, Adv. Math. 228 (2011) 97-152.

[16] Philippe Di Francesco, Integrable combinatorics, [in:] Seventeenth International Congress on Mathematical Physics, A. Jensen (ed.), pp. 29-51, World Scientific, Singapore, 2014.

[17] Aristophanes Dimakis, Folkert Müller-Hoissen, Quasi-symmetric functions and the KP hierarchy, J. Pure Appl. Algebra 214 (2010) 449-460.

[18] Adam Doliwa, Desargues maps and the Hirota-Miwa equation, Proc. R. Soc. A 466 (2010) 1177-1200.

[19] Adam Doliwa, The affine Weyl group symmetry of Desargues maps and of the noncommutative Hirota-Miwa system, Phys. Lett. A 375 (2011) 1219-1224.

[20] Adam Doliwa, Non-commutative rational Yang-Baxter maps, Lett. Math. Phys. 104 (2014) 299-309.

[21] Adam Doliwa, Rinat M. Kashaev, Non-commutative bi-rational maps satisfying Zamolodchikov equation, and Desargues lattices, J. Math. Phys. 61 (2020) 092704 (23 pp.).

[22] Adam Doliwa, Masatoshi Noumi, The Coxeter relations and KP map for noncommuting symbols, Lett. Math. Phys. 110 (2020) 2743-2762. 
[23] Gerard H. E. Duchamp, Daniel Krob, The lower central series of the free partially commutative group, Semigroup Forum 45 (1992) 385-394.

[24] Arne Dür, Möbius Functions, Incidence Algebras and Power Series Representations, Lecture Notes in Math. 1202, Springer, Berlin, 1986.

[25] Kurusch Ebrahimi-Fard, Li Guo, Dirk Kreimer, Integrable Renormalization I: the Ladder case, J.Math.Phys. 45 (2004) 3758-3769.

[26] Kurusch Ebrahimi-Fard, Li Guo, Dirk Kreimer, Integrable Renormalization II: the General case, Ann. H. Poincaré 6 (2005) 369-395.

[27] Richard Ehrenborg, On posets and Hopf algebras, Adv. Math. 119 (1996) 1-25.

[28] Pavel Etingof, Israel Gelfand, Vladimir Retakh, Nonabelian integrable systems, quasideterminants, and Marchenko lemma Math. Res. Lett. 5 (1998) 1-12.

[29] Loïc Foissy, Les algébres de Hopf des arbres enracinés décorés. I, II, Bull. Sci. Math. 126 (2002) no. 3, 193-239, no. 4, 249-298.

[30] Loïc Foissy, Faà di Bruno subalgebras of the Hopf algebra of planar tees from combinatorial Dyson-Schwinger equations, Adv. Math. 218 (2007) 136-162.

[31] Sergey Fomin, Anatol N. Kirillov, The Yang-Baxter equation, symmetric functions, and Schubert polynomials, Discrete Math. 153 (1996) 123-143.

[32] Israel M. Gelfand, Daniel Krob, Alain Lascoux, Bernard Leclerc, Vladimir R. Retakh, Jean-Yves Thibon, Noncommutative symmetric functions, Adv. in Math.112 (1995) $218-348$.

[33] Ira Gessel, Multipartite P-partitions and inner products of skew Schur functions, Contemp. Math. 34 (1984) 289-301.

[34] Darli Grinberg, Victor Reiner, Hopf algebras in combinatorics, arXiv:1409.8356.

[35] Robert Grossman, Richard G. Larson, Hopf-algebraic structures of families of trees, J. Algebra 126 (1989) 184-210.

[36] Mathieu Guay-Paquet, John Harnad, 2D Toda $\tau$-functions as combinatorial generating functions, Lett. Math. Phys. 105 (2015) 827-852.

[37] Ryogo Hirota, Discrete analogue of a generalized Toda equation, J. Phys. Soc. Jpn. 50 (1981) 3785-3791.

[38] Michael E. Hoffman, Quasi-shuffle products, J. Algebraic Combin. 11 (2000) 49-68

[39] Michael E. Hoffman, (Non)commutative Hopf algebras of trees and (quasi)symmetric functions, [in:] Renormalization and Galois theories, pp. 209-227, IRMA Lect. Math. Theor. Phys., 15, Eur. Math. Soc., Zürich, 2009.

[40] Ralf Holtkamp, Comparison of Hopf algebras on trees, Arch. Math. (Basel) 80 (2003) 368-383.

[41] Samuel K. Hsiao, A semigroup approach to wreath-product extensions of Solomon's descent algebras, Electron. J. Combin. 16 (2009) 21, 9 pp.

[42] Samuel K. Hsiao, T. Kyle Petersen, Colored posets and colored quasi-symmetric functions, Ann. Comb. 14 (2010) 251-289. 
[43] Saj-Nicole A. Joni, Gian-Carlo Rota, Coalgebra and bialgebra in combinatorics, Stud. Appl. Math. 61 (1979) 93-139.

[44] Anatol N. Kirillov, Nikolai Yu. Reshetikhin, The Yangians, Bethe ansatz and combinatorics, Lett. Math. Phys. 12 (1986) 199-208.

[45] Anatoli Klimyk, Konrad Schmüdgen, Quantum Groups and Their Representations, Springer, 1997, Berlin.

[46] Sotiris Konstantinou-Rizos, Theodoros E. Kouloukas, A noncommutative discrete potential KdV lift, J. Math. Phys. 59 (2018) 063506.

[47] Vladimir E. Korepin, Nikolay M. Bogoliubov, Anatoly G. Izergin, Quantum inverse scattering method and correlation functions, Cambridge University Press, Cambridge, 1993.

[48] Atsuo Kuniba, Tomoki Nakanishi, Junji Suzuki, T-systems and Y-systems in integrable systems, J. Phys. A: Math. Theor. 44 (2011) 103001, 146 pp.

[49] Boris A. Kupershmidt, KP or mKP: Noncommutative Mathematics of Lagrangian, Hamiltonian, and Integrable Systems, AMS, Providence, 2000.

[50] M. Lothaire, Combinatorics on words, Cambridge University Press, 1997.

[51] Kurt Luoto, Stefan Mykytiuk, Stephanie van Willigenburg, An introduction to quasisymmetric Schur functions, Springer, 2013.

[52] Ian G. Macdonald, Symmetric functions and Hall polynomials, Oxford University Press, Oxford, 1995.

[53] Percy A. MacMahon, Combinatory analysis, University Press, 1915, Cambridge.

[54] Jacques Sakarovitch, Elements of Automata Theory, Cambridge University Press, Cambridge, 2009.

[55] Shahn Majid, Foundations of Quantum Group Theory, University Press, 1995, Cambridge.

[56] Clauda Malvenuto, Christophe Reutenauer, Duality between quasi-symmetric functions and the Solomon descent algebra, J. Algebra 177 (1995) 967-982.

[57] Roberto Mantaci, Christophe Reutenauer, A generalization of Solomon's algebra for hypooctahedral groups and other wreath products, Comm. Algebra 23 (1995) 27-56.

[58] Sarah K. Mason, Recent Trends in Quasisymmetric Functions [in:] Recent Trends in Algebraic Combinatorics, H. Barcelo, G. Karaali, R. Orellana, eds., pp. 239-279, Association for Women in Mathematics Series 16, Springer, 2019.

[59] Antoni Mazurkiewicz, Introduction to trace theory, [in:] The Book of Traces, V. Diekert, G. Rozenberg, eds., pp. 3-41, World Scientific, 1995, Singapore.

[60] John W. Milnor, John C. Moore, On the structure of Hopf algebras, Ann. Math. 81 (1965) 211-264.

[61] Tetsuji Miwa, On Hirota's difference equations, Proc. Japan Acad. 58 (1982) 9-12. 
[62] Tetsuji Miwa, Michio Jimbo, Etsuro Date, Solitons: Differential equations, symmetries and infinite dimensional algebras, Cambridge University Press, Cambridge, 2000.

[63] Jonathan J. C. Nimmo, On a non-Abelian Hirota-Miwa equation, J. Phys. A: Math. Gen. 39 (2006) 5053-5065.

[64] Jean-Christophe Novelli, Jean-Yves Thibon, A one-parameter family of dendriform identities, J. Combin. Theory Ser. A 116 (2009) 864-874.

[65] Jean-Christophe Novelli, Jean-Yves Thibon, Free quasi-symmetric functions and descent algebras for wreath products, and noncommutative multi-symmetric functions, Discrete Mathematics 310 (2010) 3584-3606.

[66] Stéphane Poirier, Cycle type and descent set in wreath products, Discrete Math. 180 (1998) 315-343.

[67] Christophe Reutenauer, Free Lie Algebras, Clarendon Press, Oxford, 1993.

[68] Arto Salomaa, Matti Soittola, Automata-Theoretic Aspects of Formal Power Series, Springer, 1978, New York.

[69] Mikio Sato, Soliton equations as dynamical systems on infinite dimensional Grassmann manifolds, [in:] Random systems and dynamical systems (Kyoto, 1981), RIMS Kokyuroku 439, Kyoto Univ. (1981) 30-46.

[70] William R. Schmitt, Incidence Hopf algebras, J. Pure Appl. Algebra 96 (1994) 299330.

[71] Richard P. Stanley, Ordered structures and partitions, Mem. Amer. Math. Soc. 119, Harvard University, 1972.

[72] Richard P. Stanley, Enumerative Combinatorics, Vol. 2, Cambridge University Press, 2001, Cambridge.

[73] Thomas A. Sudkamp, Languages and Machines. An Introduction to the Theory of Computer Science, Addison Wesley Longman, Inc., 1997.

[74] Moss E. Sweedler, Hopf algebras, Benjamin, New York, 1969.

[75] Mitsuhiro Takeuchi, Free Hopf algebras generated by coalgebras, J. Math. Soc. Japan 23 (1971) 561-582.

[76] Gérard Xavier Viennot, Heaps of pieces, I; basic definitions and combinatorial lemmas, [in:] Combinatoire Énumérative, G. Labelle, P. Leroux (eds.), pp. 321-350, Springer Lecture Notes in Math. 1234, Berlin, 1986.

[77] Wenhua Zhao, A noncommuative symmetric system over the Grossman-Larson Hopf algebra of labeled rooted trees, J. Algebr. Comb. 28 (2008) 235-260.

[78] Paul Zinn-Justin, Integrability and combintorics: selected topics, [in:] Exact Methods in Low-Dimensional Statistical Physics and Quantum Computing, Lecture Notes of the Les Houches Summer School, Vol. 89, J. Jacobsen, L. F. Cugliandolo, V. Pasquier, S. Ouvry, D. Serban (eds.), pp. 483-525, Oxford University Press, 2010. 